\title{
Modelling of confined vortex rings
}

\author{
Ionut Danaila $^{1}$, Felix Kaplanski ${ }^{2}$, and Sergei Sazhin ${ }^{3}$ \\ ${ }^{1}$ Laboratoire de Mathématiques Raphaël Salem, Université de Rouen, F-76801 \\ Saint-Étienne-du-Rouvray, France \\ ${ }^{2}$ Laboratory of Multiphase Media Physics, Tallinn University of Technology, Akad. tee 15A, \\ Tallinn 12618, Estonia \\ ${ }^{3}$ Sir Harry Ricardo Laboratories, School of Computing, Engineering and Mathematics, \\ University of Brighton, Brighton BN2 4GJ, U.K.
}

(Received Received 24 October 2014; revised 31 March 2015; accepted 30 April 2015)

This paper is focused on the investigation of vortex rings evolving in a tube. A new theoretical model for a confined axisymmetric vortex ring is developed. The predictions of this model are shown to be in agreement with available experimental data and numerical simulations. The model combines the viscous vortex ring model, developed by Kaplanski and Rudi (Kaplanski \& Rudi 2005), with Brasseur's (Brasseur 1979) approach to derive a wall-induced streamfunction correction. Using the power-law assumption for the time variation of the viscous length of the vortex ring, the time variations of the main integral characteristics, circulation, kinetic energy and translational velocity, are obtained. Direct numerical simulations (DNS) are used to test the range of applicability of the model and to investigate new physical features of confined vortex rings recently reported in the experimental study by Stewart et al. (2012). The model is shown to lead to a very good approximation of the spatial distribution of the Stokes streamfunction, obtained by DNS. The vortex signature and the time evolution of the energy of the vortex are also accurately predicted by the model. A procedure for fitting the model with realistic vortex rings, obtained by DNS, is suggested. This opens the way to using the model for practical engineering applications.

\section{CONTENTS}

1. Introduction

2. A model for a viscous vortex ring in a tube

2.1. Governing equations

2.2. Brasseur's approach to modelling a confined vortex ring 5

2.3. A new model for a confined vortex ring 6

3. Direct numerical simulations of a viscous vortex ring in a tube 10

3.1. Numerical model for a vortex ring generator 10

3.2. Numerical results $\quad 12$

4. Comparison between DNS data and predictions of the model 17

4.1. Comparison algorithm 18

4.2. Streamfunction field of the confined vortex ring for $R e_{\mathrm{D}}=1700 \quad 19$

4.3. Evolution of integral characteristics for $R e_{\mathrm{D}}=1700 \quad 23$

4.4. Evolution of integral characteristics for higher Reynolds numbers $\quad 25$

$\begin{array}{ll}\text { 5. Summary and discussion } & 27\end{array}$ 


\section{Introduction}

The properties of vortex rings in unbounded domains have been studied for over a century, both theoretically and experimentally (see the reviews by Shariff \& Leonard 1992; Lim \& Nickels 1995; Fukumoto 2010). Recently, vortex ring models were used to predict the evolution of complex flows, such as those encountered in biological propulsion (Mohseni 2006; Dabiri 2009) and during fuel injection in internal combustion engines (Begg et al. 2009; Kaplanski et al. 2010; Sazhin 2014). These and other applications led to growing interest in developing simplified analytical models of vortex rings, the predictions of which are close to those inferred from the numerical analysis and experimental observations.

The mathematical theory of inviscid axisymmetric vortex rings was developed in the 70s and early 80s, mainly in connection with Hill's spherical vortex ring (Hill 1894), which is a fundamental solution of the Euler system of equations (see also Batchelor 1988; Saffman 1992). The existence and uniqueness of the solutions to these equations were studied by Fraenkel \& Berger (1974), Ni (1980), and Amick \& Fraenkel (1988) for the general case and by Amick \& Fraenkel (1986), and Norbury (1972) for vortex rings bifurcating from Hill's vortex. Based on this theory, Norbury (1973) calculated numerically, using finite difference methods, a family of inviscid vortex rings identified by a single geometric parameter. Vortex ring solutions were given in the form of tabulated Fourier series coefficients which allowed him to reconstruct vortex ring geometry, vorticity and streamline distributions. This restricted the applicability of this approach to the analysis of the observed vortex rings. Also, the model was based on the assumption that the vorticity distribution in the vortex core is linear, $i$. e. proportional to the distance to the axis of symmetry (as in Hill's spherical vortex model), while the Gaussian vorticity distribution has been observed in experiments (e.g. Weigand \& Gharib 1997; Cater et al. 2004). Despite this non-realistic assumption, the Norbury model proved useful in estimating integral characteristics and global properties of vortex rings, such as the formation number (Mohseni \& Gharib 1998; Shusser \& Gharib 2000; Linden \& Turner 2001).

Much effort has been focused on the development of more realistic modelling of vortex rings, taking into account the effects of viscosity. Tung \& Ting (1967) and Saffman (1992) developed asymptotic theories for the initial stage of the motion of an axisymmetric vortex ring, when the core radius $L$ was small compared to the initial outer radius $R_{0}$. Using these theories, a formula for the translational velocity of such a ring was derived by Saffman (1992) based on the assumption of the Gaussian distribution of vorticity and using the Helmholtz-Lamb (Helmholtz 1958; Lamb 1932) formula. Saffman's formula was generalised by Fukumoto \& Moffatt $(2000,2008)$ for rings at very large Reynolds numbers. On the other hand, Rott \& Cantwell $(1993 a, b)$ studied viscous vortex ring behaviour at large times. Using Phillips' self-similar solution to the Stokes equation for a dipole flow (Phillips 1956) and the Helmholtz-Lamb formula, these authors obtained an expression for the asymptotic drift velocity of a 'fat' vortex at the decaying stage for $L \gg R_{0}$. Later, Saffman suggested a second formula for the translational velocity of a vortex ring for the intermediate stage when $L \approx R_{0}$, which predicted results close to those obtained by Rott and Cantwell in the long-time limit (see also Weigand \& Gharib (1997)).

Recently, Kaplanski \& Rudi (2005) developed an unconfined vortex ring model based on a linear first-order solution to the Navier-Stokes equation for the axisymmetric geometry and arbitrary times (see also Kaplanski \& Rudi (1999)). The vorticity in the vortex core was predicted to be Gaussian, which makes the model more attractive for realistic 
applications. This class of vortex rings was described using a single geometric parameter which allows us to consider them as the viscous analogue to Norbury's vortices. The model is valid not only in the limit of small Reynolds numbers $R e=\Gamma_{0} / \nu$, where $\Gamma_{0}$ is the circulation of the vortex ring and $\nu$ the fluid kinematic viscosity, but also for large $R e$ at short times $t$ (Fukumoto \& Kaplanski 2008). The translational velocity of the vortex ring, obtained via the substitution of this solution into the Helmholtz-Lamb formula, was presented in a closed form in terms of the generalised hypergeometric functions. This velocity was shown to coincide with the one predicted by Saffman's formula (Fukumoto \& Kaplanski 2008) in the short-time limit and with the one predicted by Rott and Cantwel's formula in the long-time limit. In a number of papers, including those by Fukumoto \& Kaplanski (2008), Danaila \& Helie (2008), and Kaplanski et al. (2010), it was shown that the integral characteristics, such as translational velocity $U$ and energy $E$, predicted by the model, are very weak functions of the Reynolds number for all times. This opened the way of applying the model to fit numerically generated vortex rings (Danaila \& Helie 2008) or experimentally observed vortex ring flows including those observed in internal combustion engines (Begg et al. 2009; Kaplanski et al. 2010).

Radially confined vortex ring flows have been far less studied compared with unconfined vortex rings, despite their importance for many practical applications, ranging from vortex ring-like structures in gasoline engines (Begg et al. 2009) to transient flow fields in biological complex systems (Gharib et al. 2006). Recent experimental study by Stewart et al. (2012) demonstrated important differences between the evolution of a vortex ring confined in a tube and its evolution in an unbounded domain. For example, higher decay rates of circulation were observed for vortex rings confined in a tube, compared with unconfined ones. The first attempt, and the only one to the best of our knowledge, to perform theoretical analysis of confined vortex rings was made by Brasseur (1979). This author considered an axisymmetric vortex ring confined in an infinitely long tube and assumed that the flow field in the outer region (far from the core) depends on the ring's total circulation, but not on the details of the vorticity distribution in the vortex core. This hypothesis allowed him to model the streamfunction of the confined vortex ring as the sum of an unconfined streamfunction for a circular vortex filament (Lamb 1932) and the one induced by the tube. One of the limitations of the model suggested by Brasseur (1979) is that it is based on a rather simplistic theoretical model for a vortex ring, the predictions of which cannot be easily matched with realistic results of numerical simulations or experimental observations.

The main goal of the present paper is to suggest a new model for a viscous vortex ring placed into a radially confined domain. This model will be based on the generalisation of the analytical developments by Brasseur (1979), using the recent viscous vortex ring model suggested by Kaplanski \& Rudi (2005). Direct numerical simulations will be used to assess the quality of the model predictions, with particular emphasis on the confinement effects.

In $\S 2$ we present basic equations for a radially confined vortex ring and derivation of their approximate analytical solution, combining Brasseur's approach and the vortex ring model suggested by Kaplanski \& Rudi (2005). Results of numerical simulations of the vortex ring evolution in a tube are presented in $\S 3$. These will be used to describe the physics of the flow, using flow parameters inferred from experimental data provided by Stewart et al. (2012). Extensive comparisons between theoretical predictions of the model and direct numerical simulation (DNS) data are presented in $\S 4$. The main contributions of the paper are summarised in $\S 5$. 


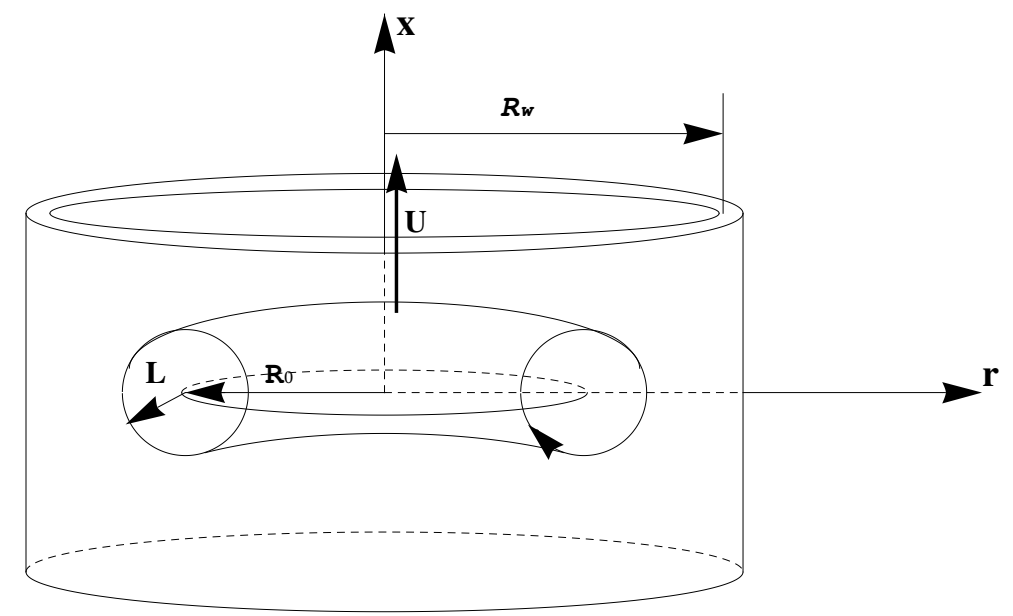

FiguRE 1. Schematic of a vortex ring in a tube.

\section{A model for a viscous vortex ring in a tube}

\subsection{Governing equations}

We consider an axisymmetric vortex ring of radius $R_{0}$ placed in an infinitely long rigid tube of radius $R_{w}$ (see figure 1), moving in an incompressible fluid with translational velocity $U$ along the axis of symmetry $(x)$. The governing equations for the vorticity $(\omega)$ and streamfunction $(\Psi)$ can be presented as (Batchelor 1988):

$$
\begin{gathered}
\frac{\partial \omega}{\partial t}+\frac{\partial}{\partial r}\left(-\frac{1}{r} \frac{\partial \Psi}{\partial x} \omega\right)+\frac{\partial}{\partial x}\left(\frac{1}{r} \frac{\partial \Psi}{\partial r} \omega\right)=\nu\left(\frac{\partial^{2} \omega}{\partial r^{2}}+\frac{\partial^{2} \omega}{\partial x^{2}}+\frac{1}{r} \frac{\partial \omega}{\partial r}-\frac{\omega}{r^{2}}\right), \\
\mathcal{L} \Psi=\omega, \quad \mathcal{L}:=-\frac{\partial}{\partial r}\left(\frac{1}{r} \frac{\partial}{\partial r}\right)-\frac{\partial}{\partial x}\left(\frac{1}{r} \frac{\partial}{\partial x}\right),
\end{gathered}
$$

where $x, r$ are the axes of a cylindrical coordinate system and $t$ is time. Note that $\mathcal{L}$ is a linear, self-adjoint elliptic operator. We consider the following boundary conditions: symmetry at the axis:

$$
\omega=\Psi=0, \quad \text { for } \quad r=0,
$$

vanishing vorticity and streamfunction in the far field of the vortex:

$$
\omega, \Psi \rightarrow 0 \quad \text { when } \quad\left(x^{2}+r^{2}\right)^{1 / 2} \rightarrow \infty, \text { for } r<R_{w},
$$

and no flow through the tube wall:

$$
\omega \rightarrow 0, \quad \frac{1}{r} \frac{\partial \Psi}{\partial x}=0, \quad \text { for } \quad r=R_{w} .
$$

Our vortex ring model will be developed using equations (2.1)-(2.2) with boundary conditions (2.3)-(2.5). Note that boundary condition (2.5) can be used for vortex rings in a tube as long as the viscous interaction of the rings with the tube wall remains weak and can be ignored (see also Brasseur (1979)). For longer time instants, when this assumption is not valid, the boundary condition (2.5) should be replaced with a non-slip wall condition. Note also that direct numerical simulations in $\S 3$ will use a velocitypressure formulation of the Navier-Stokes equations, with non-slip boundary conditions at the tube wall. 


\subsection{Brasseur's approach to modelling a confined vortex ring}

Brasseur (1979) modelled a confined vortex ring of circulation $\Gamma_{0}$ and radius $R_{0}$ (see figure 1) assuming that in the region $r>R_{0}$ the velocity potential $\Phi_{c}$ is equal to the sum $\Phi_{c}=\Phi+\Phi_{0}$, where $\Phi$ is the potential of a circular vortex filament (CVF) in an unbounded flow:

$$
\Phi=-\frac{\Gamma_{0} R_{0}}{2} \int_{0}^{\infty} \exp (-x \mu) \mathrm{J}_{1}\left(R_{0} \mu\right) \mathrm{J}_{0}(r \mu) d \mu
$$

and $\Phi_{0}$ is the velocity potential added to $\Phi$ to satisfy the boundary condition (2.5) of no flow through the tube wall. The velocity potential $\Phi$ for a circular vortex filament corresponds to the streamfunction (Lamb 1932)

$$
\Psi=\frac{\Gamma_{0} R_{0} r}{2} \int_{0}^{\infty} \exp (-x \mu) \mathrm{J}_{1}\left(R_{0} \mu\right) \mathrm{J}_{1}(r \mu) d \mu,
$$

where $\mathrm{J}_{1}$ is the Bessel function of the first kind. Note that formula (2.7) is valid for $x>0$ and the sign of $\Psi$ is opposite to the one given in Lamb (1932).

Potential (2.6) can be expanded into a series of harmonic poles at the origin (Brasseur 1979):

$$
\Phi(\tilde{r}, \tilde{x})=\frac{\varepsilon \Gamma_{0}}{2}\left(\frac{\varepsilon}{2} \frac{\partial}{\partial \tilde{x}}-\frac{\varepsilon^{3}}{2^{2} 4} \frac{\partial^{3}}{\partial \tilde{x}^{3}}+\frac{\varepsilon^{5}}{2^{2} 4^{2} 6} \frac{\partial^{5}}{\partial \tilde{x}^{5}}-\ldots\right) \int_{0}^{\infty} \exp (-\mu \tilde{x}) \mathrm{J}_{0}(\tilde{r} \mu) d \mu,
$$

where

$$
\tilde{x}=\frac{x}{R_{w}}, \quad \tilde{r}=\frac{r}{R_{w}}, \quad \varepsilon=\frac{R_{0}}{R_{w}} .
$$

The parameter $0<\varepsilon<1$ quantifies the confinement of the vortex ring. When $\varepsilon \rightarrow 0$, the first harmonic can be simplified:

$$
\int_{0}^{\infty} \exp (-\mu \tilde{x}) \mathrm{J}_{0}(\tilde{r} \mu) d \mu=\frac{1}{\tilde{z}}=\frac{1}{\sqrt{\tilde{x}^{2}+\tilde{r}^{2}}}
$$

and (2.8) becomes:

$$
\Phi(\tilde{r}, \tilde{x})=\frac{\varepsilon \Gamma_{0}}{2}\left(\frac{\varepsilon}{2} \frac{\partial}{\partial \tilde{x}}\left(\frac{1}{\tilde{z}}\right)-\frac{\varepsilon^{3}}{2^{2} 4} \frac{\partial^{3}}{\partial \tilde{x}^{3}}\left(\frac{1}{\tilde{z}}\right)+\frac{\varepsilon^{5}}{2^{2} 4^{2} 6} \frac{\partial^{5}}{\partial \tilde{x}^{5}}\left(\frac{1}{\tilde{z}}\right)-\ldots\right) .
$$

The first harmonic (2.10) shows that at infinity the vortex ring looks like a point dipole; the contributions of other terms in (2.11) are found as the derivatives with respect to $\tilde{x}$.

Brasseur (1979) replaced the expression of the first harmonic (2.10) with a monopole at the origin in a tube and then derived the velocity potential $\Phi_{0}$ and the corresponding streamfunction $\Psi_{0}$ induced by the presence of the tube:

$$
\begin{gathered}
\Phi_{0}=-\frac{\Gamma_{0} R_{0}}{\pi} \int_{0}^{\infty} \frac{\mathrm{K}_{1}\left(\mu R_{w}\right)}{\mathrm{I}_{1}\left(\mu R_{w}\right)} \mathrm{I}_{1}\left(R_{0} \mu\right) \mathrm{I}_{0}(r \mu) \sin (x \mu) d \mu, \\
\Psi_{0}=\frac{\Gamma_{0} R_{0} r}{\pi} \int_{0}^{\infty} \frac{\mathrm{K}_{1}\left(\mu R_{w}\right)}{\mathrm{I}_{1}\left(\mu R_{w}\right)} \mathrm{I}_{1}\left(R_{0} \mu\right) \mathrm{I}_{1}(r \mu) \cos (x \mu) d \mu,
\end{gathered}
$$

where $K_{1}$ is the modified Bessel function of the second kind.

Finally, the streamfunction corresponding to a confined vortex ring can be expressed as

$$
\Psi_{c}=\Psi-\Psi_{0},
$$

where $\Psi$ is the streamfunction (2.7) of a CVF in an unbounded flow and $\Psi_{0}$ is the streamfunction (2.13) induced by the presence of the tube. 


\subsection{A new model for a confined vortex ring}

Brasseur (1979) modelled a vortex ring as a circular vortex filament (CVF) in a bounded flow. In a new model, described in this section, we apply Brasseur's approach but replace the vortex filament with the more realistic vortex ring model suggested by Kaplanski \& Rudi (2005).

We start by recalling the main features of the vortex ring model developed in Kaplanski \& Rudi (1999, 2005). Introducing local coordinates (see figure 1)

$$
\sigma=r / L, \quad \eta=\left(x-X_{c}\right) / L, \quad \theta=R_{0} / L,
$$

where $X_{c}$ is the axial coordinate of the vortex centre and $L$ is the diffusivity scale of the ring's core, the following expressions were obtained for vorticity

$$
\omega=\frac{\Gamma_{0}}{\sqrt{2 \pi}} \frac{\theta^{3}}{R_{0}^{2}} \exp \left(-\frac{\sigma^{2}+\eta^{2}+\theta^{2}}{2}\right) \mathrm{I}_{1}(\sigma \theta),
$$

circulation

$$
\Gamma=\Gamma_{0}\left\{1-\exp \left(-\frac{\theta^{2}}{2}\right)\right\}
$$

kinetic energy

$$
E=\frac{\Gamma_{0}^{2} R_{0} \theta}{2}\left[\frac{1}{12} \sqrt{\pi} \theta_{2}^{2} \mathrm{~F}_{2}\left(\left\{\frac{3}{2}, \frac{3}{2}\right\},\left\{\frac{5}{2}, 3\right\},-\theta^{2}\right)\right],
$$

translational velocity

$$
\begin{gathered}
U=\frac{\Gamma_{0} \theta \sqrt{\pi}}{4 \pi R_{0}}\left[3 \exp \left(-\frac{\theta^{2}}{2}\right) \mathrm{I}_{1}\left(\frac{\theta^{2}}{2}\right)+\frac{\theta^{2}}{12}{ }_{2} \mathrm{~F}_{2}\left(\left\{\frac{3}{2}, \frac{3}{2}\right\},\left\{\frac{5}{2}, 3\right\},-\theta^{2}\right)\right. \\
\left.-\frac{3 \theta^{2}}{5}{ }_{2} \mathrm{~F}_{2}\left(\left\{\frac{3}{2}, \frac{5}{2}\right\},\left\{2, \frac{7}{2}\right\},-\theta^{2}\right)\right],
\end{gathered}
$$

and streamfunction

$$
\Psi_{\mathrm{VR}}=\frac{\Gamma_{0} R_{0} \sigma}{4} \int_{0}^{\infty}\left[\exp (\eta \mu) \operatorname{erfc}\left(\frac{\mu+\eta}{\sqrt{2}}\right)+\exp (-\eta \mu) \operatorname{erfc}\left(\frac{\mu-\eta}{\sqrt{2}}\right)\right] \mathrm{J}_{1}(\theta \mu) \mathrm{J}_{1}(\sigma \mu) d \mu,
$$

where

$$
\operatorname{erfc}(x)=\frac{2}{\sqrt{\pi}} \int_{x}^{\infty} \exp \left(-t^{2}\right) d t
$$

$\mathrm{J}_{1}$ is the Bessel function of the first kind, $\mathrm{I}_{1}$ is the modified Bessel function of the first kind and ${ }_{2} \mathrm{~F}_{2}$ is the generalised hypergeometric function.

Equations (2.16)-(2.19) were originally derived for $L=\sqrt{2 \nu t}$, i. e. laminar vortex ring. Later it was shown that they remain valid in a more general case, when $L$ is approximated as $a t^{b}$, where $a$ and $b$ are constants $(1 / 4 \leqslant b \leqslant 1 / 2)$ (Kaplanski et al. 2009). This generalised vortex ring model was successfully applied to the analysis of vortex rings observed in gasoline internal combustion engines (Begg et al. 2009; Kaplanski et al. 2010).

At the initial stage when the core is very thin $\nu t \ll R_{0}^{2}(\theta \gg 1)$, the model describes Saffman's early-time vortex ring with outer radius $R_{0}$ (Saffman 1970). At this stage the Gaussian form of the vorticity field is strongly compressed and the vorticity isocontours $\omega / \omega_{\max }$ are circular (Kaplanski et al. 2012). At the decaying stage $\nu t \gg R_{0}{ }^{2}(\theta \ll 1)$, the model predicts the Stokes-dipole solution (Rott \& Cantwell 1993a). For these cases, all characteristics of vortex rings, including kinetic energy (2.18) and translational velocity (2.19), at short and long times are given by the closed-form asymptotic expressions and 
are identical to the well-known Saffman's and Rott-Cantwell formulae, respectively. The Gaussian distribution of vorticity predicted by equation (2.16) at short times agrees with experimental observations of vortex rings (Weigand \& Gharib 1997; Cater et al. 2004), although this distribution does not follow from a rigorous solution to the nonlinear Navier-Stokes equation.

To apply the same approach as Brasseur (1979), we need to rescale the vortex ring model using the following variables instead of those in (2.15):

$$
x_{1}=\frac{x-X_{c}}{R_{0}}=\frac{\eta}{\theta}, \quad r_{1}=\frac{r}{R_{0}}=\frac{\sigma}{\theta} .
$$

In this case the dimensionless vorticity and streamfunction are defined as:

$$
\omega^{*}=\frac{\omega R_{0}^{2}}{\Gamma_{0}}, \Psi_{\mathrm{vR}}^{*}=\frac{\Psi_{\mathrm{VR}}}{\Gamma_{0} R_{0}} .
$$

We recall that the Reynolds number of the flow was introduced as $R e=\Gamma_{0} / \nu$ and the parameter $\varepsilon=R_{0} / R_{w}<1$ quantifies the confinement of the vortex ring. The wall is located at $r_{1 w}=1 / \varepsilon$.

We restrict our analysis to the time interval in which a vortex ring has a well defined maximum in its vorticity distribution, and is located far from the wall of the tube (small $\varepsilon$ ). The parameter $\theta$ (depending on the diffusivity length) is then defined as:

$$
\theta=\frac{R_{0}}{\sqrt{2 \nu t}}
$$

Thus, the non-dimensional time can be expressed as:

$$
t^{*}=\frac{\nu t}{R_{0}^{2}}=\frac{1}{2 \theta^{2}} .
$$

Having substituted (2.22) and (2.24) into (2.16) we obtain the following expression for the non-dimensional vorticity (cf. Fukumoto \& Kaplanski 2008):

$$
\omega^{*}=\frac{\theta^{3}}{\sqrt{2 \pi}} \exp \left(-\frac{\left(r_{1}^{2}+x_{1}^{2}+1\right) \theta^{2}}{2}\right) \mathrm{I}_{1}\left(r_{1} \theta^{2}\right) .
$$

The expression for the non-dimensional streamfunction follows from (2.20):

$\Psi_{\mathrm{VR}}^{*}=\frac{r_{1} \theta}{4} \int_{0}^{\infty}\left[\exp \left(x_{1} \theta \mu\right) \operatorname{erfc}\left(\frac{\mu+x_{1} \theta}{\sqrt{2}}\right)+\exp \left(-x_{1} \theta \mu\right) \operatorname{erfc}\left(\frac{\mu-x_{1} \theta}{\sqrt{2}}\right)\right] \mathrm{J}_{1}\left(r_{1} \theta \mu\right) \mathrm{J}_{1}(\theta \mu) d \mu$.

Using the leading term of the expansion for $z_{1}=\theta \sqrt{x_{1}^{2}+r_{1}^{2}} \rightarrow \infty$, we can obtain an approximation of the streamfunction (2.27) at a large distance from the ring's core:

$$
\begin{aligned}
& \Psi_{\mathrm{VR}}^{*} \quad \approx \frac{r_{1} \theta}{4} \int_{0}^{\infty}\left[2 \exp \left(-\left|x_{1}\right| \theta \mu\right)+\exp \left(-z_{1}^{2} / 2\right) \mathcal{O}\left(\frac{1}{z_{1}^{2}}\right)\right] \mathrm{J}_{1}\left(r_{1} \theta \mu\right) \mathrm{J}_{1}(\theta \mu) d \mu \\
& \approx \frac{r_{1} \theta}{2} \int_{0}^{\infty} \exp \left(-\left|x_{1}\right| \theta \mu\right) \mathrm{J}_{1}(\theta \mu) \mathrm{J}_{1}\left(r_{1} \theta \mu\right) d \mu=\frac{r}{2} \int_{0}^{\infty} \exp (-|x| \mu) \mathrm{J}_{1}\left(R_{0} \mu\right) \mathrm{J}_{1}(r \mu) d(2.28)
\end{aligned}
$$

Note that the approximation of the streamfunction by expression (2.28) is valid for positive and negative values of $x_{1}$ and is identical to expression (2.7). Thus expression (2.28) describes the streamfunction of a circular filament of vorticity. Therefore, it is expected that the vortex structure, which diffuses with time and transforms into a 'fat' vortex ring, has the same large-distance asymptotics as a circular line vortex, identical to Brasseur's CVF. 

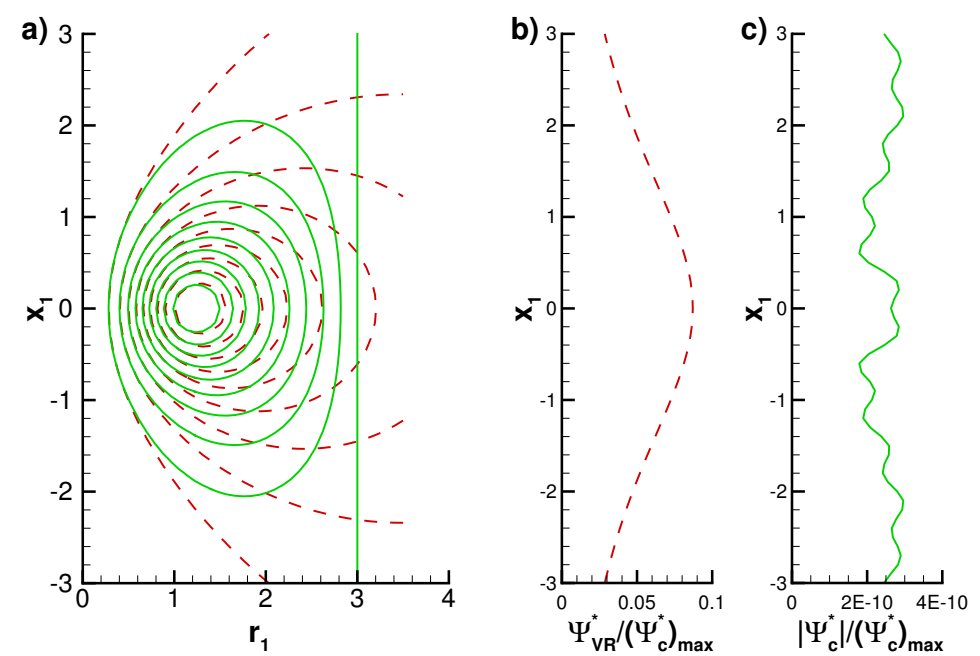

FIgURE 2. (a) Isocontours of the normalised streamfunctions $\Psi_{c}^{*} /\left(\Psi_{c}^{*}\right)_{\max }$ for a confined ring, predicted by (2.29) for $\varepsilon=1 / 3, \theta=3$ (solid curves), and $\Psi_{\mathrm{VR}}^{*} /\left(\Psi_{\mathrm{VR}}^{*}\right)_{\max }$ for an unbounded ring with $\theta=3$ (see expression $(2.27)$ ) (dashed curves). Contours are shown for $\Psi_{c}^{*} /\left(\Psi_{c}^{*}\right)_{\max }$ from 0.1 to 0.9 with an increment of 0.1 . The vertical line at $r_{1}=3$ represents the tube wall for the confined ring. Profiles along the tube wall line $\left(r_{1}=3\right)$ for $\Psi_{\mathrm{VR}}^{*}(\mathrm{~b})$ and $\left|\Psi_{c}^{*}\right|(\mathrm{c})$.

Based on this observation, we can find the streamfunction describing the flow generated by a vortex ring in a tube, following the approach suggested by Brasseur (1979) (see Expression (2.14)). The dimensionless streamfunction satisfying the boundary conditions (2.3)-(2.5) is presented as:

$$
\Psi_{c}^{*}\left(x_{1}, r_{1}\right)=\Psi_{\mathrm{VR}}^{*}\left(x_{1}, r_{1}\right)-\frac{r_{1}}{\pi} \int_{0}^{\infty} \frac{\mathrm{K}_{1}(\mu / \varepsilon)}{\mathrm{I}_{1}(\mu / \varepsilon)} \mathrm{I}_{1}(\mu) \mathrm{I}_{1}\left(r_{1} \mu\right) \cos \left(x_{1} \mu\right) d \mu,
$$

where $\Psi_{\mathrm{VR}}^{*}$ is given by $(2.27)$.

Expression (2.29) shows that at large distances, Expression (2.27) transforms into Brasseur's CVF. Thus at even larger distances this expression will have the same asymptotics as Brasseur's CVF in the form of the first term of (2.8) (asymptotics for a dipole). Brasseur used this feature and changed asymptotics at large distances to satisfy boundary condition $(1 / r) \partial \Psi / \partial x=0$ at $r=R_{w}$. As our solution at large distances is identical to Brasseur's CVF, we expect that the additional streamfunction (2.13) induced by the presence of the tube will act on the combined streamfunction in the same way as described by Brasseur. To illustrate the deformation of the streamlines in the vicinity of the wall, in figure 2 we plot the contours of $\Psi_{c}^{*}$ given by (2.29) and compare them with the streamlines $\Psi_{\mathrm{VR}}^{*}$ (given by equation $(2.27)$ ) for the vortex ring in an unbounded domain. Recalling that in the region $r_{1}<1 / \varepsilon$ there are no discontinuities, expression (2.29) was calculated using the software package Mathematica, Version 6 ((Mathematica 2007)). It was assumed that $\theta=3$ and $\varepsilon=1 / 3$. As one can clearly see from figures 2 (b) and (c) displaying streamfunction profiles along the wall boundary $\left(r_{1}=r_{1 w}=1 / \varepsilon\right)$, $\Psi_{c}^{*}$ remains close to zero (and thus satisfies the boundary condition (2.5)) within the machine-precision error. $\Psi_{\mathrm{VR}}^{*}$ reaches the maximum value at $x_{1}=0$, which is one order of magnitude below $\left(\Psi_{c}^{*}\right)_{\max }$; consequently, the wall correction at this point takes its largest value to bring $\Psi_{c}^{*}$ to zero.

To assess the range of applicability of our model, we estimate below the accuracy of the approximation (2.28) by computing the maximum error at the tube wall. Following 


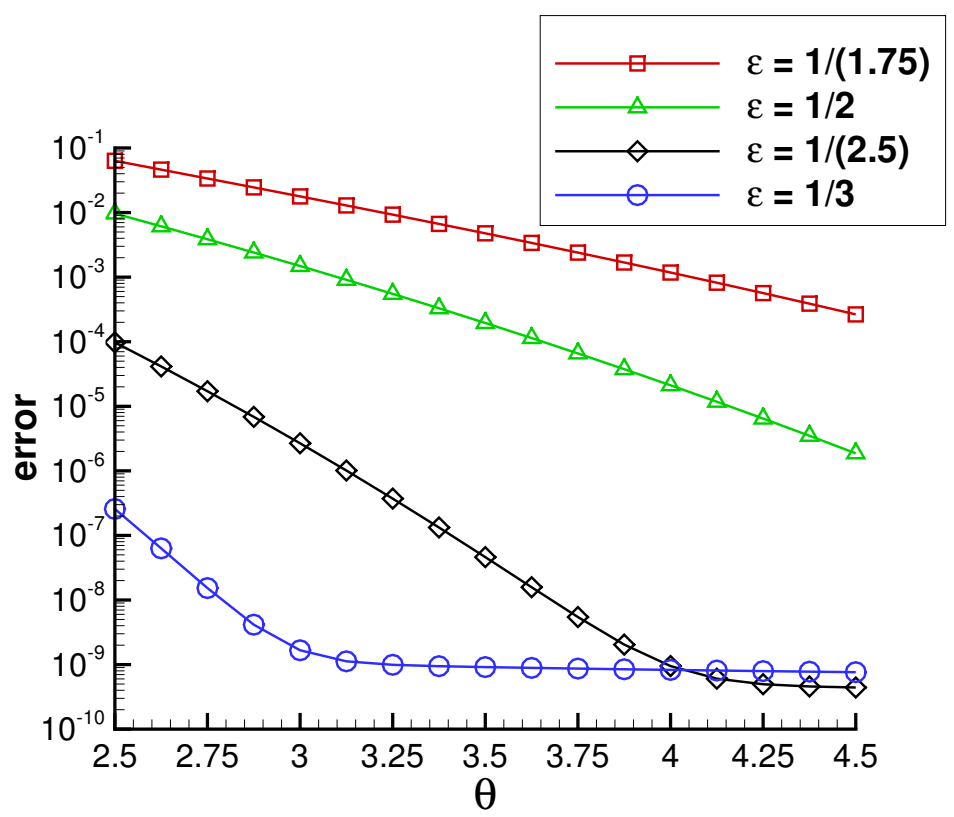

Figure 3. Maximum relative error of the model defined as the ratio $\Psi_{c}^{*}\left(x_{1}=0, r_{1}=1 / \varepsilon\right) /\left(\Psi_{c}^{*}\right)_{\max }$, as a function of the parameter $\theta$ characterising the vortex thickness (see expression 2.15). The values of this error were computed for various confinement parameters $\varepsilon$ using the confined vortex ring model (2.29).

figure 2 (b) and (c), the most important correction occurs at $r=R_{w}\left(r_{1}=1 / \varepsilon\right)$ for $x_{1}=0$ (corresponding to the vortex centre). This can be theoretically justified by using symmetry considerations (Esteban 1983) and the maximum principle (Fraenkel \& Berger 1974). We therefore define the maximum relative error of the model as

$$
\text { error }=\frac{\Psi_{c}^{*}\left(x_{1}=0, r_{1}=1 / \varepsilon\right)}{\left(\Psi_{c}^{*}\right)_{\max }}=\frac{\Psi_{c}^{*}\left(x_{1}=0, r_{1}=1 / \varepsilon\right)}{\Psi_{c}^{*}\left(x_{1}=0, r_{1}=1\right)},
$$

where we have used the fact that the maximum value of the streamfunction occurs at the centre of the vortex $\left(x_{1}=0, r_{1}=1\right)$.

Figure 3 shows the maximum relative error of the model for various confinement parameters $\varepsilon$ and for a range of the parameter $\theta$ relative to the values obtained from DNS (see next sections). We recall that parameter $\theta$, defined by $(2.24)$, is a measure of the thickness of the vortex ring (see figure 1 ). The considered values $(2.5 \leqslant \theta \leqslant 4.5)$ correspond to thin vortex rings and are typical of experimentally or numerically generated vortex rings. The maximum error does not exceed $10 \%$ for the strongest confinement $(\varepsilon=1 /(1.75))$ and the thickest vortex $\operatorname{ring}(\theta=2.5)$. For $\varepsilon \geqslant 1 / 2$, the errors are negligible for the whole range of parameter $\theta$. For typical values most relevant to practical applications (Danaila \& Helie 2008; Fukumoto 2010), $3 \leqslant \theta \leqslant 4.5$, and our confined vortex ring model can be applied with negligible errors for all confinement parameters. 


\section{Direct numerical simulations of a viscous vortex ring in a tube}

In this section, we investigate some features of the flow in confined vortex rings using the numerical code developed by Danaila et al. (see Danaila \& Helie (2008); Danaila et al. (2009)) for simulation of axisymmetric vortex rings. Some of these features, not observed in previous studies of unconfined vortex rings, were identified in recent experiments by Stewart et al. (2012).

As in the previous section, we consider an axisymmetric vortex ring placed in an infinitely long rigid tube of radius $R_{w}$ (see figure 1), moving along the axis of symmetry ( $x$-axis) in an incompressible fluid. The incompressible Navier-Stokes equations at cylindrical coordinates $(r, \theta, x)$ were solved using the numerical method suggested by Rai \& Moin (1991) and modified by Verzicco \& Orlandi (1996). The method is described in detail by Orlandi (1999) (see also Ruith et al. (2004)). The equations were written in primitive variables $\left(r \cdot v_{r}, v_{\theta}, v_{x}\right)$ and solved on a staggered grid to avoid the problem of singularities at the axis $r=0$. Second order finite differences were used for the spatial discretisation. For time advancement we used the fractional-step method described by Kim \& Moin (1985). This method is based on a combination of a low-storage third-order Runge-Kutta scheme for the convective terms and the semi-implicit Crank-Nicolson scheme for the viscous terms. At each substep of the Runge-Kutta scheme, the momentum equations were solved by an approximate factorisation technique (see Kim \& Moin (1985)) and the Poisson equation was solved for the pressure correction. The Poisson solver uses a fast Fourier transformation following the azimuthal direction $\theta$ and an effective cyclic reduction method (Fishpack subroutines) for solving the remaining two-dimensional system following $(r, x)$ directions. The method is globally second order accurate in space and time.

\subsection{Numerical model for a vortex ring generator}

Laminar vortex rings are usually produced in laboratories by pushing a column of fluid (by a piston/cylinder mechanism, e.g. Gharib et al. (1998), or a piston gun, e.g. Sullivan et al. (2008)) through a long pipe of diameter $D$. In what follows, all parameters are normalised using $D$ as the length-scale and the maximum piston velocity $U_{0}$ as the velocity scale. The main physical parameter of the flow is the Reynolds number based on the pipe diameter:

$$
R e_{\mathrm{D}}=\frac{U_{0} D}{\nu}
$$

Using the time scale $t_{0}=D / U_{0}$, the non-dimensional time in simulations is defined as:

$$
\tau=\frac{t U_{0}}{D}=R e_{\mathrm{D}}\left(\frac{R_{0}}{D}\right)^{2} t^{*}
$$

where $t^{*}$ is the non-dimensional time defined by (2.25) (the same time was used in the theoretical model).

The vortex ring is generated numerically by prescribing an appropriate axial velocity profile at the outlet section of the pipe, corresponding to the inlet section of the computational domain. For this purpose, we used the specified discharge velocity (SDV) model suggested by Danaila et al. (2009):

$$
U_{\mathrm{SDV}}(r, \tau)=U_{0} U_{p}(\tau) U_{\mathrm{CL}}(\tau) U_{b}(r, \tau)
$$

where $U_{p}(\tau)$ is the piston velocity program (normalised by its maximum value $\left.U_{0}\right), U_{\mathrm{CL}}(\tau)$ takes into account the time evolution of the centreline velocity, and $U_{b}(r, \tau)$ describes the radial dependence of the profile. 
The SDV model is based on the analysis of the Stokes flow evolution inside a long axisymmetric pipe with unitary constant velocity profile at the entry section. At the exit of the pipe, the centreline velocity evolves in time as:

$$
U_{\mathrm{CL}}(\tau)=\frac{1}{1-\frac{8}{\sqrt{\pi R e_{\mathrm{D}}}} \sqrt{\tau}+\frac{8}{R e_{\mathrm{D}}} \tau} .
$$

The time evolution of the momentum thickness $\Theta$ and discharge jet radius $R_{j e t}$ are given as:

where

$$
\Theta(\tau)=\frac{\sqrt{2}-1}{\sqrt{\pi}} B(\tau), \quad R_{\text {jet }}(\tau)=\frac{1}{2}-0.477 B(\tau),
$$

$$
B(\tau)=\frac{2}{\sqrt{\operatorname{Re}_{\mathrm{D}}}} \sqrt{\tau}
$$

To describe the radial dependence of the axial velocity profile, the hyperbolic tangent velocity profile (e.g. Michalke (1984)) commonly used in jet-flow simulations, was modified to account for the time evolution of its main characteristics:

$$
U_{b}(r, \tau)=\frac{1}{2}\left\{1+\tanh \left[\frac{1}{4 \Theta(\tau)}\left(1-\frac{r}{R_{\text {jet }}(\tau)}\right)\right]\right\} .
$$

Note that in most numerical simulations (e.g. Zhao et al. (2000); James \& Madnia (1996)), the parameters of the discharge velocity profile are assumed to be constant: $U_{\mathrm{CL}}=1, R_{\text {jet }}=0.5$ and $\Theta=\Theta_{0}$ is specified (to prescribe a fixed vorticity layer thickness). This SDV model proved to be very accurate in simulating the laminar vortex ring evolution (see Danaila et al. (2009)), when compared to available experimental data.

For the piston velocity program we used the model suggested by Zhao et al. (2000):

$$
U_{p}(\tau)= \begin{cases}\frac{1}{2}\left[1-\cos \left(\pi \frac{\tau}{\tau_{1}}\right)\right], & \tau \leqslant \tau_{1} \\ \frac{1}{2}\left[1+\cos \left(\pi \frac{\tau-\tau_{1}}{\tau_{2}-\tau_{1}}\right)\right], & \tau_{1}<\tau \leqslant \tau_{2} .\end{cases}
$$

Using the results of recent experiments described by Stewart et al. (2012) the following fitting constants were obtained (see fig. 4): $\tau_{1}=1.57$ and $\tau_{2}=2.26$.

The main integral characteristics of the vortex generator can be approximately estimated by the so-called slug-model (Shariff \& Leonard 1992; Lim \& Nickels 1995). This model assumes that at the exit plane of the vortex generator the flow is parallel to the axis and the rate of change of the circulation is determined by the vorticity flux across this plane. Thus, the stroke length $\left(L_{p}\right)$, circulation $\left(\Gamma_{p}\right)$, impulse $\left(I_{p}\right)$ and kinetic energy $\left(E_{p}\right)$ of the discharged fluid at the cylinder tip are calculated as:

$$
\begin{gathered}
L_{p}=\int_{0}^{\tau_{\text {off }}} U_{\mathrm{CL}}(\tau) d \tau, \quad \Gamma_{p}=\frac{1}{2} \int_{0}^{\tau_{\text {off }}} U_{\mathrm{CL}}^{2}(\tau) d \tau, \\
I_{p}=\frac{\pi D^{2}}{4} \int_{0}^{\tau_{\text {off }}} U_{\mathrm{CL}}^{2}(\tau) d \tau, E_{p}=\frac{\pi D^{2}}{8} \int_{0}^{\tau_{\text {off }}} U_{\mathrm{CL}}^{3}(\tau) d \tau .
\end{gathered}
$$

Taking $R e_{\mathrm{D}}=1770$ and $\tau_{\text {off }}=2.26$, as in the experiments by Stewart et al. (2012), we obtain from (3.4) and (3.9) the following values: $L_{p}=1.28, \Gamma_{p}=0.55, I_{p}=0.86$, $E_{p}=0.41$.

Note that the stroke ratio $\left(L_{p}=1.28\right)$ is lower than the so-called formation number of the vortex ring, reported to be in the range 3.6 to 4.5 (Gharib et al. 1998; Krueger 


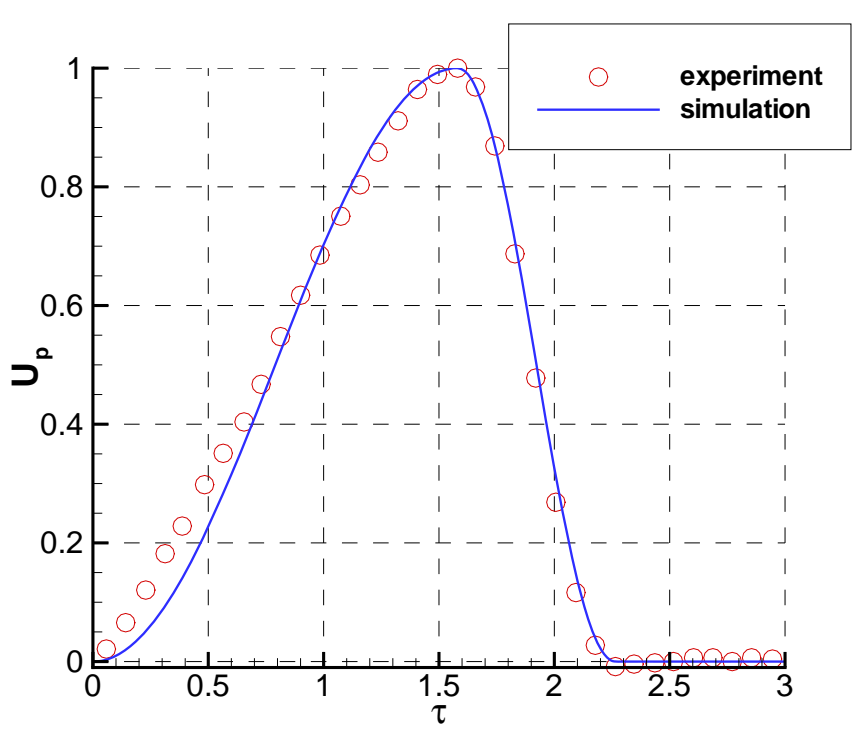

Figure 4. Piston velocity $U_{p}(\tau)$ used for numerical simulations (solid curve) and experimentally observed piston velocity reported by Stewart et al. (2012) (symbols).

\& Gharib 2003; Dabiri \& Gharib 2005). This implies that all the vorticity produced by the vortex generator is expected to be engulfed into the vortex ring, $i . e$. the separation (pinch-off) between the vortex ring and its tail, observed for stroke ratios exceeding 4, is not expected to be present for this case.

\subsection{Numerical results}

We use the same computational setting as in Danaila \& Helie (2008) and in Danaila et al. (2009) as in these studies, the long time evolution of a laminar vortex ring was accurately captured. The length of the computational domain was taken as $L_{d}=10$, which allowed us to avoid the vortex ring coming close to the downstream boundary, where we apply the convective boundary condition (Orlanski 1976), enforced by the global mass conservation procedure suggested by Ruith et al. (2004). At $r=R_{w}$ a non-slip boundary condition is imposed through a ghost-cell procedure. The grid was uniform in both axial and radial directions with a refined grid size $\delta r=\delta x=0.01$ which ensures grid convergence of the results. Tests with a stretched grid in the radial direction, such that at least 30 grid points are clustered in the vorticity layer at the inflow, showed identical results. The timestep was set to $\delta \tau=0.001$, which is largely below the admissible value imposed by the stability of the numerical scheme. Timestep refinement tests showed negligible differences in the results obtained for lower $\delta \tau$.

To investigate the physics of a confined vortex ring, we first fix the confinement parameter and assume that $D_{w} / D=1.75$, as inferred from the experiments by Stewart et al. (2012). Note that this confinement parameter is more appropriate to describe experiments or numerical simulations than the parameter $\varepsilon=D_{0} / D_{w}$ defined in the theoretical analysis. Indeed, the value of the diameter of the vortex ring $D_{0}$ (see figure 1) cannot be specified after prescribing the injection parameters. The two parameters are related by the formula $D_{w} / D=D_{0} /(\varepsilon D)$; a very rough approximation of the latter relation leads to the following expression: $D_{w} / D \approx 1 / \varepsilon$. 

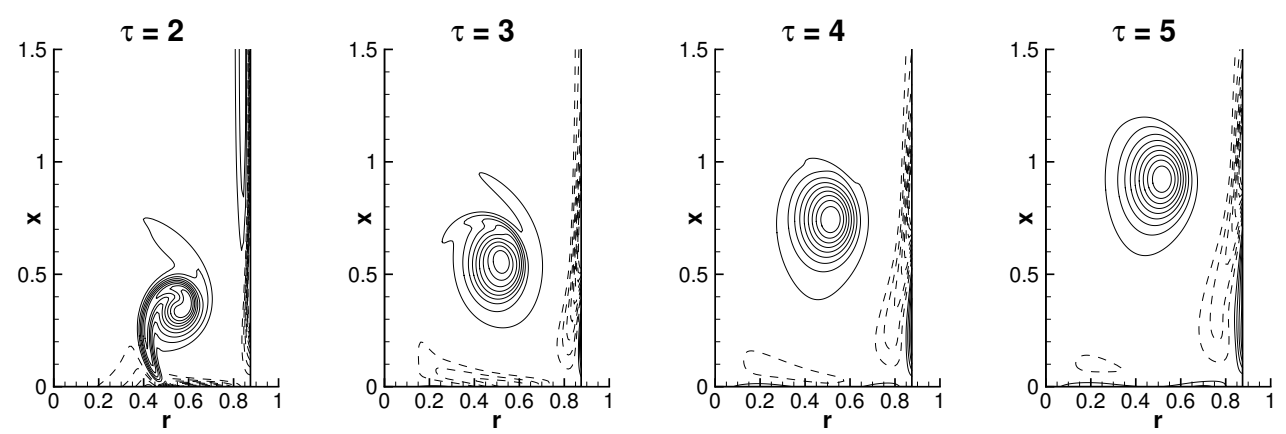

Figure 5. Contours of vorticity $\omega$ at the early stage of formation of the vortex ring in a tube. Dashed curves show contours of negative vorticity. Time instants $\tau=2,3,4,5$ are shown above the plots; $R e_{\mathrm{D}}=1700, D_{w} / D=1.75$.

The early stages of the vortex ring formation are illustrated by contours of vorticity in figure 5. Due to a very small piston stroke ratio, a vortex forms rapidly near the inlet section. The vortex ring evolves near the lateral wall, generating a negative vorticity layer that interacts with the stopping vortex, which is produced at the inlet section after the injection stops $\left(\tau>\tau_{\text {off }}=2.26\right)$. Note that we did not model the termination of the injection process, since our analysis referred to the cases when injections stopped suddenly. The stopping vortex normally travels back into the vortex generator and does not interact with the vortex ring (see the discussion by Krueger (2008)).

The influence of the confinement parameter $D_{w} / D$ on the vortex ring evolution is illustrated in figure 6 . During the injection phase $\left(\tau \leqslant \tau_{\text {off }}=2.26\right)$ the formation of the vortex ring is not affected by the proximity of the wall. This was also observed in the experimental study by Stewart et al. (2012). After the injection stops, the vortex ring moves faster when $D_{w} / D$ increases, i.e. the confinement is reduced (see figure 6 for $\tau=5$ and $\tau=10$ ). This is attributed to the interaction of the vortex ring with the vorticity layer (of the opposite sign) induced at the wall (the details of the wall vorticity are visible in figure 5 for $\tau=5$ ). Also, one can observe from figure 6 that the influence of confinement becomes negligible for large $D_{w} / D$. Our simulations showed that for $D_{w} / D \geqslant 3$, the lateral wall does not produce significant impact on the vortex ring evolution. This observation is also supported by the analysis of the time evolution of the parameters characterising the vortex ring strength and trajectory: the maximum value of the vorticity $\omega_{\max }$ (figure $7 \mathrm{a}$ ) and the stream-wise position $X_{c}$ (figure $7 \mathrm{~b}$ ) of the vortex centre corresponding to the maximum vorticity. As expected, the value of $\omega_{\max }$ for the vortex ring becomes larger and the ring travels faster when the confinement is reduced $\left(D_{w} / D\right.$ is increased). For $D_{w} / D \geqslant 3$ the results are very close to the case $D_{w} / D=8$, which can represent the asymptotic regime of an unconfined vortex ring. We can conclude that the influence of the lateral wall is negligible for $D_{w} / D \geqslant 3$, which is consistent with previously reported experimental findings (Stewart et al. 2012).

The main finding by Stewart et al. (2012) was that the circulation of the vortex ring during the post-formation phase rapidly decreases in the presence of the lateral wall. To compute vortex ring circulation, the vortex core must be properly separated from the background flow. Usually, the centre $C$ of the vortex is first located as the point of the maximum vorticity $\omega_{\max }$; the vortex core is then defined as the inner domain bounded by the vorticity contour $\omega / \omega_{\max }=0.05$ that encircles the centre $C$. The cutoff level is set based on trial and error, to the best satisfaction of the authors. Threshold values 

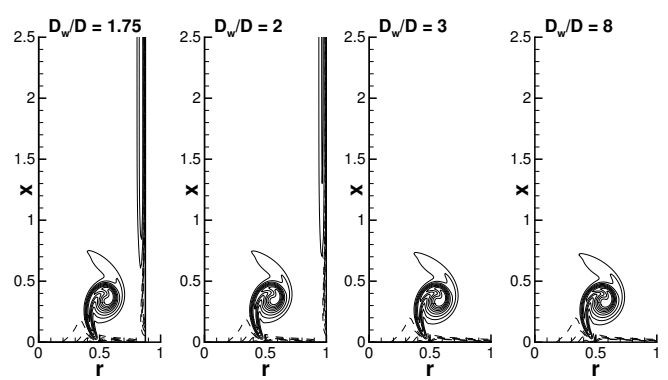

a) $\tau=2$
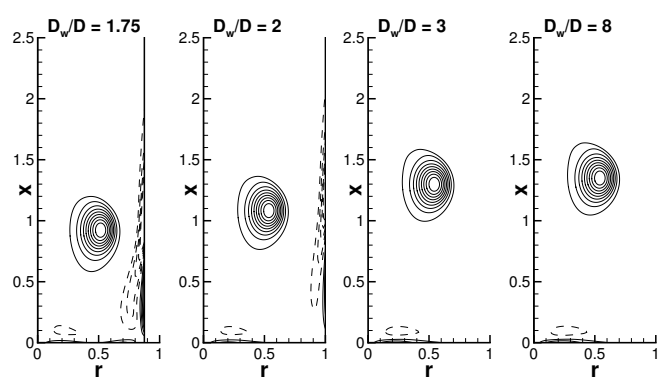

b) $\tau=5$
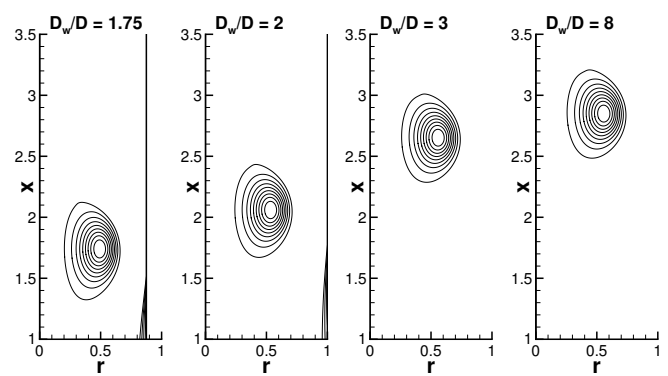

c) $\tau=10$

FiguRE 6 . Contours of the normalised vorticity $\omega / \omega_{\max }$ at various time instants and confinement parameters: $D_{w} / D=1.75,2,3,8 ; R e_{\mathrm{D}}=1700$. The values of vorticity increase from -0.3 to 0.9 with increments of 0.1 ; dashed curves show contours of negative vorticity.

ranged from 2\% (Mohseni 2001) and 5\% (Rosenfeld et al. 1998; Zhao et al. 2000; Sau \& Mahesh 2007) in numerical studies, to approximately $10 \%$ (Dabiri \& Gharib 2004) in experiments.

The presence of the lateral wall makes the separation of the vortex core rather delicate, since intense vorticity layers develop near this wall and at the inflow section after the injection stops. A special post-processing program, based on the free finite element solver FreeFem++ (Hecht et al. 2007; Hecht 2012), was designed to properly separate the vortex ring core and compute flow integrals. The program identifies all closed contours for the prescribed threshold of $\omega / \omega_{\max }$ (see figure 8), selects the unique contour around the vortex ring centre and then meshes this domain with triangles. All flow characteristics are then represented on the finite element mesh using quadratic $P_{2}$ triangular elements. The interpolation between the finite difference grid (used for DNS computations) and the finite element mesh is also quadratic, which is consistent with the spatial accuracy of the Navier-Stokes solver. The advantage of this finite element post-processing is that 

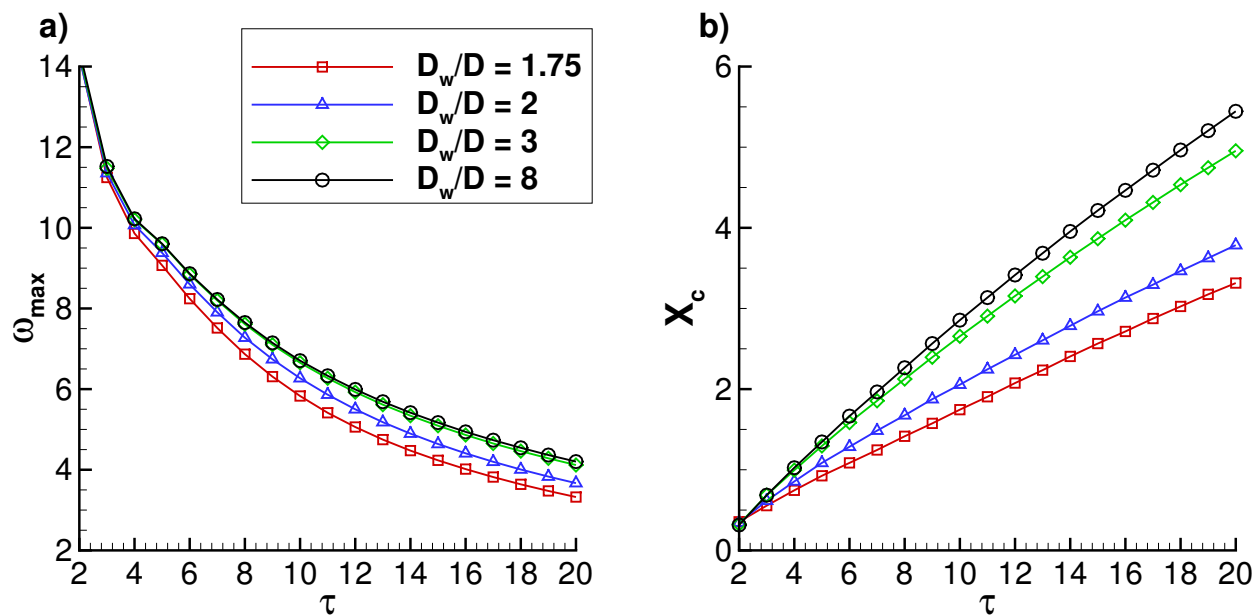

FiguRE 7 . Time evolution of maximum vorticity $\omega_{\max }$ and stream-wise position of the vorticity centre $X_{c}$ corresponding to the maximum vorticity; $R e_{\mathrm{D}}=1700$.
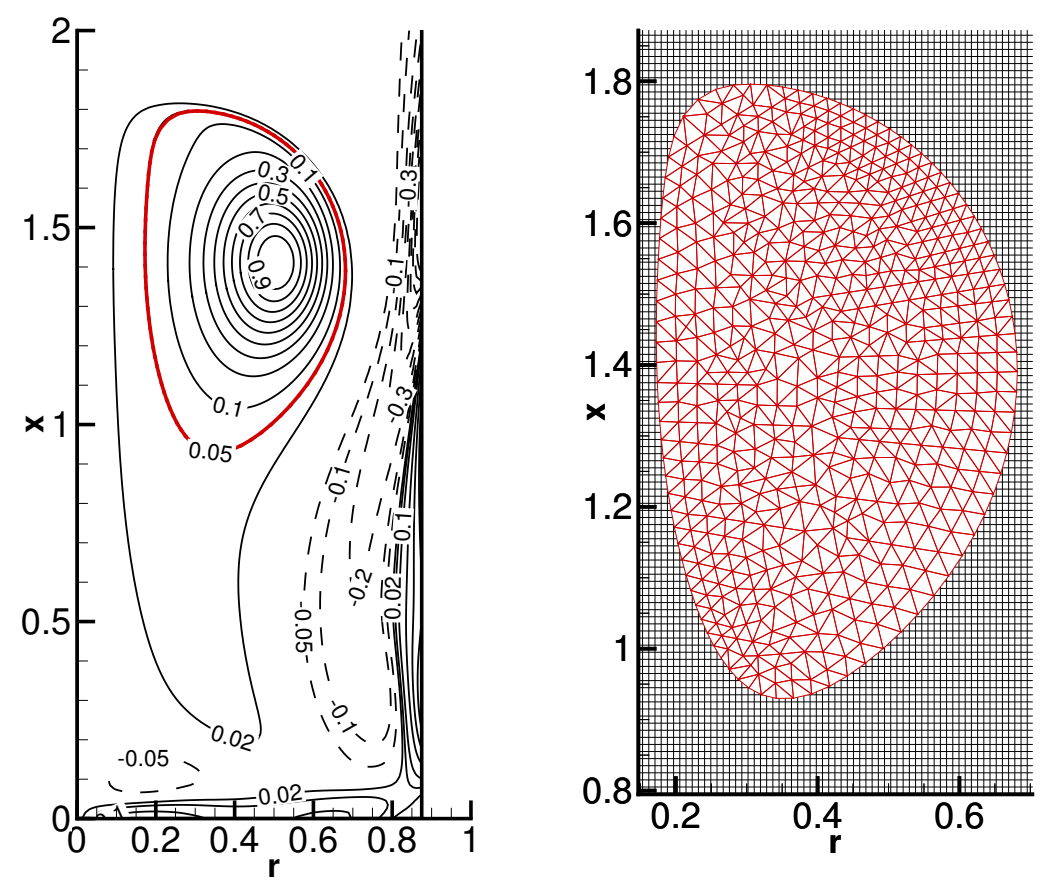

FIGURE 8. Left: contours of the normalised vorticity $\omega / \omega_{\max }$ for the vortex ring at $\tau=8$; $R e_{\mathrm{D}}=1700, D_{w} / D=1.75$. Dashed curves show contours of negative vorticity. Right: illustration of the post-processing procedure using finite elements: the contour $\omega / \omega_{\max }=0.05$ is identified and then the inside area is remeshed using triangular finite elements (this zoom into the finite element mesh using larger triangles is shown for clarity). 
a)

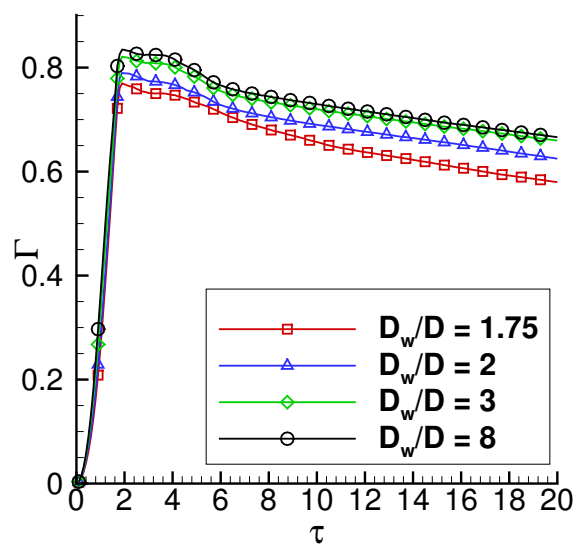

b)

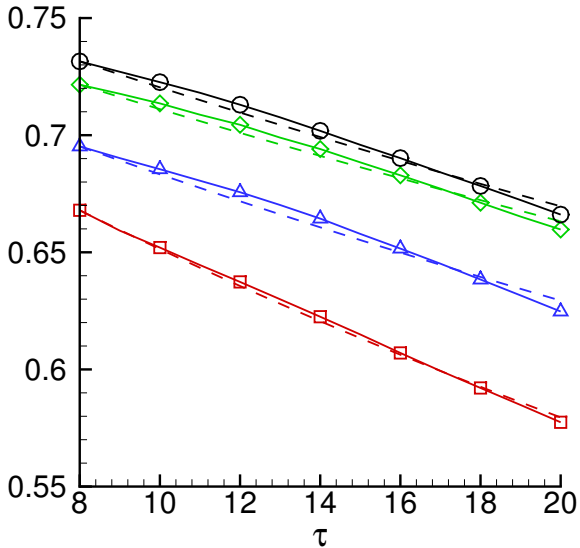

Figure 9. (a) Time evolution of the vortex ring circulation for four values of the confinement parameter $D_{w} / D$. (b) Zoom corresponding to the post-formation phase of the vortex ring evolution. Dashed curves show the results inferred from equation (3.10) suggested by Stewart et al. (2012). $R e_{\mathrm{D}}=1700$.

it enables us to represent accurately and smoothly the boundary of the vortex core and, then, to use high-order (up to seventh order) Gaussian methods to compute the integral characteristics (circulation, impulse, energy) presented in the following section. Also, this post-processing toolbox can be easily used to post-process experimental results (obtained from PIV measurements), since FreeFem ++ is an integrated product with its own high level programming language with a syntax close to mathematical formulations.

The time evolution of circulation corresponding to vortex ring cores separated using this method is shown in figure 9 (a). After rapid increase during the injection phase $\left(\tau \leqslant \tau_{\text {off }}=2.26\right)$, the circulation attains a small plateau $(2.26 \leqslant \tau \leqslant 6)$ and then starts to decrease in the post-formation phase. Assuming that the vortex ring is completely formed when the contour line $\omega / \omega_{\max }=0.05$ defines a convex set (see figure 8 ), the post-formation phase starts at $\tau=8$. A zoom of the time evolution of circulation during this phase is shown in figure $9(\mathrm{~b})$. A higher rate of decrease of the circulation is observed when the confinement is increased (the ratio $D_{w} / D$ is decreased). This is consistent with experimental observations by Stewart et al. (2012) who suggested that the time evolution of the circulation $\Gamma(\tau)$ can be described by the ordinary differential equation $d \Gamma / d \tau=-\beta \Gamma^{2}$ with the solution:

$$
\Gamma(\tau)=\frac{\Gamma_{f}}{\Gamma_{f} \beta\left(\tau-\tau_{f}\right)+1},
$$

where $\Gamma_{f}$ refers to the (fitted) circulation at $\tau=\tau_{f}\left(\Gamma_{f}\right.$ was taken as the peak circulation in the experiments). The predictions of equation (3.10), taking $\tau_{f}=8$, and shown in figure 9 (b) are in good agreement with DNS results. The fitting coefficient $\beta$, which is related to the production of vorticity at the confinement wall, decreases with increasing $D_{w} / D$, although not exponentially, as reported by Stewart et al. (2012). This could be related to the fact that our fit refers to a long time evolution during the post-formation phase, while Stewart et al. (2012) considered a very short time interval after the injection stopped. We can conclude that the model equation (3.10) can be used to describe the decrease in circulation even during the post-formation phase. 

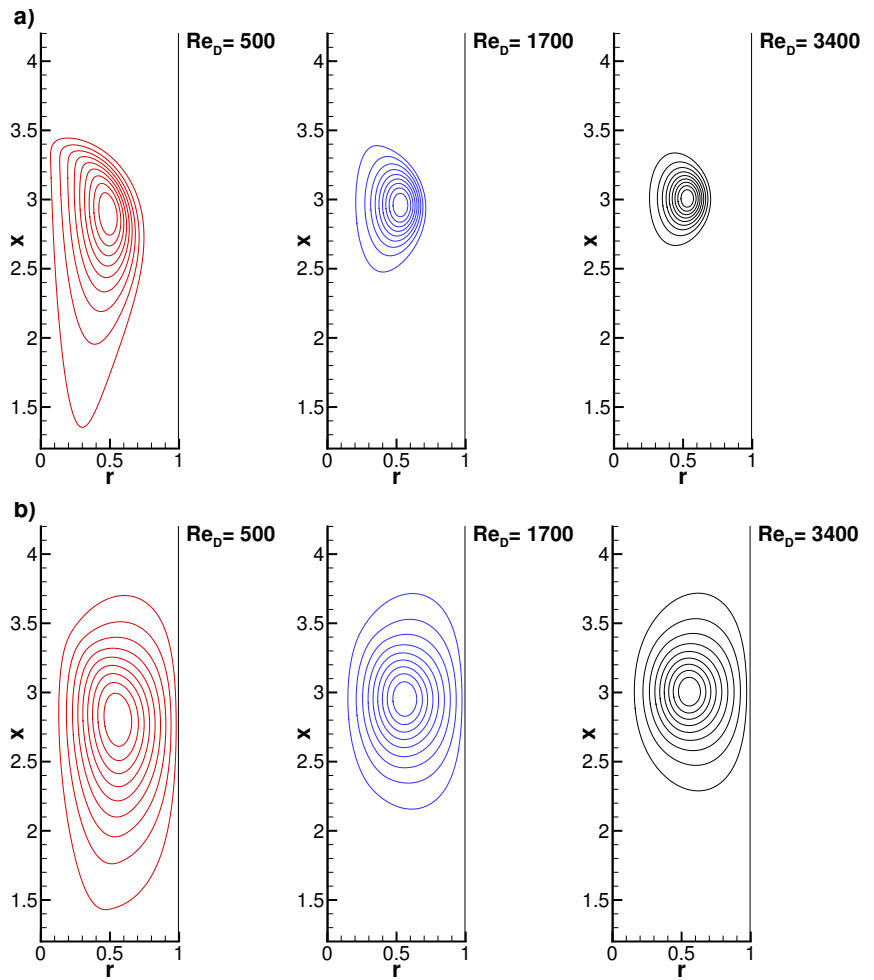

FIGURE 10. Contours of normalised vorticity $\omega / \omega_{\max }$ (a) and corresponding normalised streamfunction $\psi / \psi_{\max }$ (b) for the time instant $\tau=15$ and confinement parameter $D_{w} / D=2$; the values of $\omega / \omega_{\max }$ are shown from 0.1 to 0.9 with increments of 0.1 . $R e_{\mathrm{D}}=500,1700,3400$.

The influence of the viscous diffusion on the flow physics was investigated by performing the simulations for three Reynolds numbers. Figure 10 shows the vorticity and streamfunction distributions for the time instant $\tau=15$, confinement parameter $D_{w} / D=2$ and 3 values of $R e_{\mathrm{D}}$. For $R e_{\mathrm{D}}=500$ the vortex ring diffuses rapidly and generates large regions of opposite vorticity at the confinement wall; the post-formation phase is not clearly defined in this case, since the vortex ring almost fills the tube. For larger Reynolds numbers $\left(R e_{\mathrm{D}}=3400\right)$ the vorticity distribution looks more compact than for $R e_{\mathrm{D}}=1700$, used in previous plots and in experiments by Stewart et al. (2012). This suggests that to test our analytical model using DNS data we need to use not only the reference value $R e_{\mathrm{D}}=1700$, but also the more challenging case of higher Reynolds number $R e_{\mathrm{D}}=3400$.

\section{Comparison between DNS data and predictions of the model}

One of the attractive features of the model described in section 2 is that the closed formulae for the vorticity and streamfunction allow us to easily calculate the integral characteristics which are important in the characterisation of the flow. Since the vorticity defined by (2.26) depends on parameter $\theta$, which is a function of time (see equation (2.24)), formulae (4.3) to (4.5) can predict time evolution of integral characteristics. This allows us to trace time evolution of circulation, energy and translational velocity for a given time interval and compare model predictions with DNS data. 


\subsection{Comparison algorithm}

To enable us to compare the predictions of the model and DNS results, we assume that the pinch-off (Gharib et al. 1998; Mohseni 2001), i. e. the separation between the vortex ring and the vorticity tail, has taken place and the vortex ring is completely formed. Thus, we consider the vortex ring evolution over large time intervals during the post-formation phase.

The comparison of the predictions of the model with DNS data starts with fitting the vortex rings, obtained by DNS and the model at a given time instant $\tau=\tau_{f}$. We use equation (2.16) for the vorticity distribution, with the approximation $I_{1}(\sigma \theta) \approx$ $\exp (\sigma \theta) / \sqrt{2 \pi \sigma \theta}$, valid for large $\theta$. For the cases shown below $\theta>4$, which makes this approximation possible. Thus, we fit the DNS vorticity field with the expression:

$$
\omega_{f}=\frac{\Gamma_{f}}{\pi}\left(\frac{R_{f}}{r}\right)^{1 / 2} \frac{1}{2 L_{f}^{2}} \exp \left(-\frac{1}{2 L_{f}^{2}}\left(r-R_{0 f}\right)^{2}-\frac{1}{2 L_{f}^{2}}\left(x-X_{c f}\right)^{2}\right) .
$$

Parameters $\Gamma_{f}, R_{0 f}, X_{c f}, L_{f}$ are found using a non-linear fit (BFGS minimization method) integrated in the finite-element post-processing algorithm. We compute $\theta_{f}=R_{0 f} / L_{f}$ to complete the definition of the corresponding theoretical vortex ring. Formula (2.16) is then used to reconstruct the theoretical vorticity field $\omega(r, x)$ and compute integral characteristics in the same way as in DNS (taking into account only the vorticity of the vortex core for which $\left.\omega / \omega_{\max }>0.05\right)$.

After fitting the DNS vortex with the prediction of the model at $\tau=\tau_{f}$, we can predict the time evolution of integral characteristics assuming (see (2.25)) that

$$
L(\tau)=L_{f}\left(\frac{\tau}{\tau_{f}}\right)^{1 / 2}, \quad \tau \geqslant \tau_{f}
$$

The non-dimensional circulation $\left(\Gamma^{*}\right)$, energy $\left(E^{*}\right)$ and translational velocity $\left(U^{*}\right)$ predicted by the model are then calculated using $\omega^{*}$ given by $(2.26)$ and $\Psi_{c}^{*}$ by $(2.29)$ :

$$
\begin{gathered}
\Gamma^{*}=\frac{\Gamma}{\Gamma_{f}}=\frac{1}{\Gamma_{f}} \int_{-\infty}^{\infty} \int_{0}^{R_{w}} \omega d r d x=\int_{-\infty}^{\infty} \int_{0}^{1 / \varepsilon} \omega^{*} d r_{1} d x_{1} \\
E^{*}=\frac{E}{\Gamma_{f}^{2} R_{0 f}}=\frac{\pi}{\Gamma_{f}^{2} R_{0 f}} \int_{-\infty}^{\infty} \int_{0}^{R_{w}} \omega \Psi_{c} d r d x=\pi \int_{-\infty}^{\infty} \int_{0}^{1 / \varepsilon} \omega^{*} \Psi_{c}^{*} d r_{1} d x_{1}, \\
U^{*}=\frac{U R_{0 f}}{\Gamma_{f}}=\frac{R_{0 f}}{\Gamma_{f}} \int_{-\infty}^{\infty} \int_{0}^{R_{w}}\left(\Psi_{c}-6 x \frac{\partial \Psi_{c}}{\partial x}\right) \omega d r d x /\left(2 \int_{-\infty}^{\infty} \int_{0}^{R_{w}} r^{2} \omega d r d x\right) \\
=\frac{1}{2}\left(\int_{-\infty}^{\infty} \int_{0}^{1 / \varepsilon}\left(\Psi_{c}^{*}-6 x_{1} \frac{\partial \Psi_{c}^{*}}{\partial x_{1}}\right) \omega^{*} d r_{1} d x_{1} / \int_{-\infty}^{\infty} \int_{0}^{1 / \varepsilon} r_{1}^{2} \omega^{*} d r_{1} d x_{1}\right) .
\end{gathered}
$$

The links between non-dimensional characteristics used for the model (starred variables) and corresponding characteristics used in DNS computations (we recall that $t_{\mathrm{DNS}}=\tau$ ) are given by the following expressions:

$$
\begin{gathered}
t^{*}=\frac{1}{R e_{\mathrm{D}}}\left(\frac{D}{R_{0 f}}\right)^{2} \tau, \quad \Gamma^{*}=\frac{R e_{\mathrm{D}}}{R e} \Gamma_{\mathrm{DNS}}, \quad R e=\frac{\Gamma_{f}}{\nu} \\
\Psi^{*}=\frac{R e_{\mathrm{D}}}{R e}\left(\frac{D}{R_{0 f}}\right) \Psi_{\mathrm{DNS}}, \quad E^{*}=\left(\frac{R e_{\mathrm{D}}}{R e}\right)^{2}\left(\frac{D}{R_{0 f}}\right) E_{\mathrm{DNS}}, \quad U^{*}=\frac{R e_{\mathrm{D}}}{R e}\left(\frac{R_{0 f}}{D}\right) U_{\mathrm{DNS}}
\end{gathered}
$$




\section{a) DNS-F}

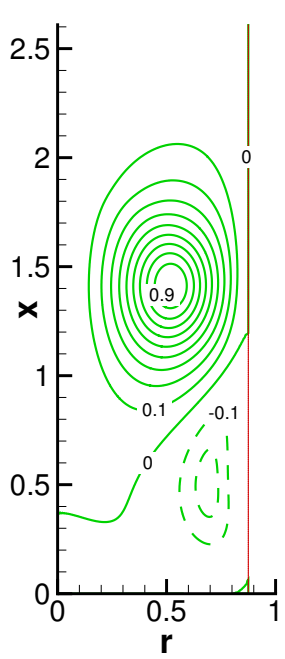

b) DNS

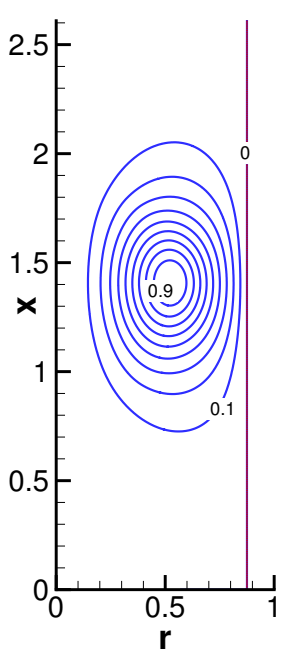

c)

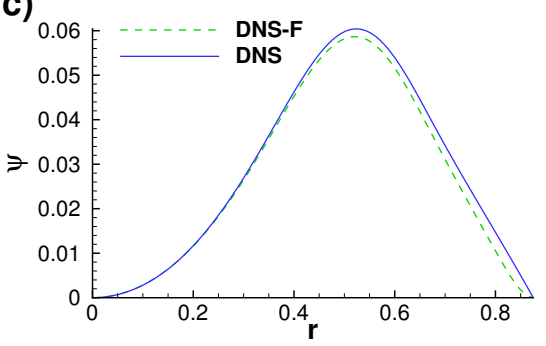

d)

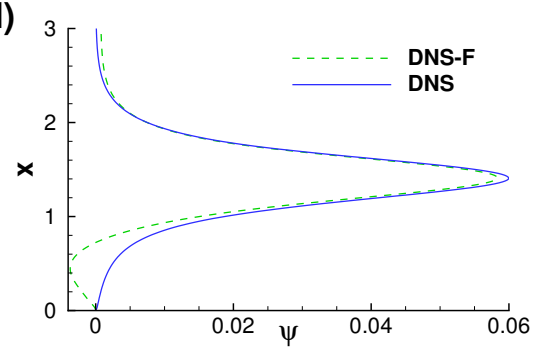

FIGURE 11. Contours of normalised streamfunction $\psi / \psi_{\max }$ obtained by solving numerically the equation (2.2) with RHS term corresponding to the full DNS vorticity field (a, DNS-F) and to the vorticity inside the vortex ring core $\left(\omega / \omega_{\max } \geqslant 0.05\right)$ (b, DNS). Streamfunction profiles through the centre of the vortex: (c) along the $r$-axis $\left(x=X_{c}\right)$ and (d) along the $x$-axis $\left(r=R_{0}\right)$. $R e_{\mathrm{D}}=1700, D_{w} / D=1.75, \tau=8$.

In what follows, all the characteristics are referred to using DNS (non-starred) variables.

Finally, the translational velocity of the vortex ring is computed from DNS data as

$$
U_{\mathrm{DNS}}=\frac{d X_{\omega c}}{d \tau}, \quad X_{\omega c}=\int_{-\infty}^{\infty} \int_{0}^{R_{w}} r^{2} x \omega d x d r / \int_{-\infty}^{\infty} \int_{0}^{R_{w}} r^{2} \omega d x d r
$$

where $X_{\omega c}$ is the streamwise coordinate of the vorticity centroid (Hill 1894; Krieg \& Mohseni 2013). This will be compared to that given by Expression (4.5).

\subsection{Streamfunction field of the confined vortex ring for $R e_{D}=1700$}

Firstly, we consider the case $R e_{\mathrm{D}}=1700, D_{w} / D=1.75$ studied in the previous section and used in the experiments described by Stewart et al. (2012). We focus on the postformation phase $(\tau \geqslant 8)$ characterised by a decay in circulation with time (see figure 9 ). We recall that for DNS data, streamfunction $\psi$ is computed by solving numerically $(2.2)$ with the vorticity corresponding to the vortex ring core $\left(\omega / \omega_{\max } \geqslant 0.05\right)$. The vorticity layer generated at the wall is thus ignored, to allow comparison with the theoretical model which does not take into account such effects. From figure 8 it can be seen that the negative vorticity at the lateral wall is rather small $\left(\left|\omega / \omega_{\max }\right| \leqslant 0.01\right)$. Its influence on the spatial distribution of the streamfunction is thus expected to be limited to the downstream part of the vortex ring. Figure 11 illustrates this statement by displaying streamfunction fields calculated using the full vorticity field (DNS-F, plot a) and the vorticity of the vortex ring core only (DNS, plot b). Streamfunction profiles through the centre of the vortex, along the $r$-axis (plot c) and $x$-axis (plot $\mathrm{d}$ ) show that differences between the two cases are negligible inside the vortex ring core. Therefore, we will use DNS streamfunction fields corresponding to the vorticity truncated to zero outside the vortex ring core. 
a)

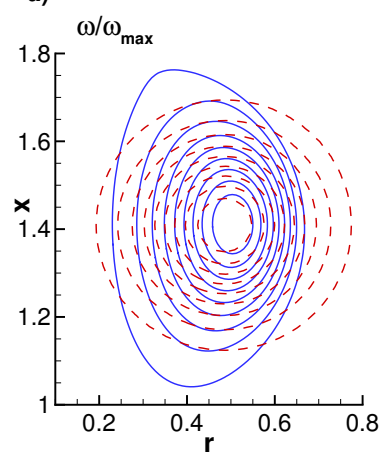

c) b)

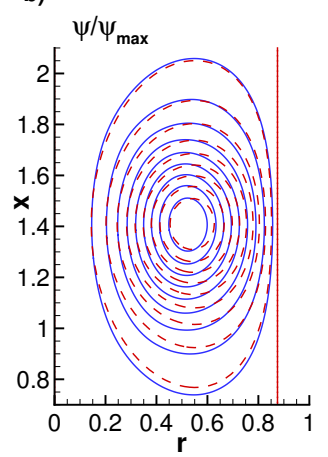

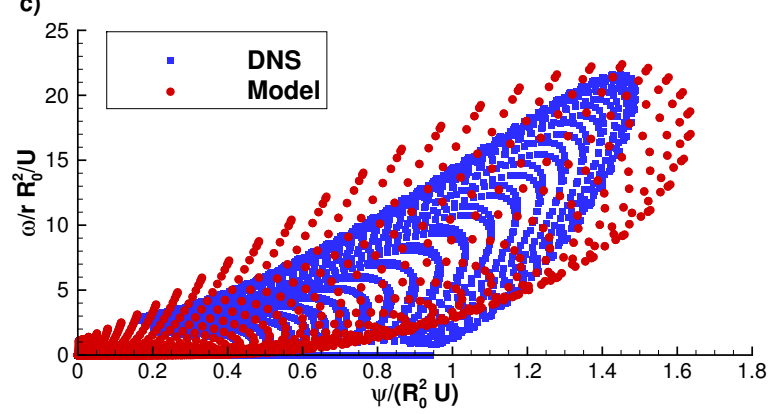

Figure 12. Comparison between the DNS data (blue solid curves or squares) and predictions of the vortex ring model (red dashed curves or circles). (a) Contours of normalised vorticity $\omega / \omega_{\max }$; (b) corresponding contours of normalised streamfunction $\psi / \psi_{\max } ;$ (c) scatter plot $\left\{\psi_{p}, \omega_{p} / r_{p}\right\}$ (nondimensional values) for points $p$ inside the vortex ring core. Values of $\omega / \omega_{\max }$ and $\psi / \psi_{\max }$ from 0.1 to 0.9 with increments of 0.1 are shown in (a) and (b). $R e_{\mathrm{D}}=1700$, $D_{w} / D=1.75, \tau=8$.

In figure 12 the results of comparison of the DNS data with the predictions of the vortex ring model for $\tau=8$ are shown. As expected, the vortex ring model (see formula (2.16) and its approximation (4.1) with an isotropic two-dimensional Gaussian) predicts quasi-circular contours for vorticity, compared to the prolate shape contours for the DNS vortex. Nevertheless, the non-linear fit provides accurate localisation of the vortex centre $\left(R_{0 f}, X_{c f}\right)$, as indicated in table 1 , part (a). Also, the circulation predicted by the vortex ring model is very close to that obtained for the DNS vortex. Note that the model for a confined vortex ring is based on the assumption that the space distribution of the vorticity is not very important in deriving Expression (2.29) for the streamfunction. This is confirmed by the results shown in figure 12 (b) demonstrating a very good agreement between DNS data and predictions of the model for the normalised streamfunction contours. Streamlines predicted by the model are almost circular close to the vortex centre, but deform near the wall to accommodate the wall boundary condition, as in the case of DNS results. The lateral wall located at $R_{w}=0.875$ is captured as a streamline in both cases.

Figures 12 (a) and (b) also suggest that the DNS vortex ring could not be accurately described by models derived as steady solutions to Euler equations (e. g. Norbury model). For inviscid and steady flows, the vorticity equation (2.1) reduces to $\omega / r=F(\Psi)$, with $F$ being an arbitrary function, $i . e . \omega / r$ propagates without change of shape along streamlines. For such models, a good theoretical representation of the streamfunction field would 
a)

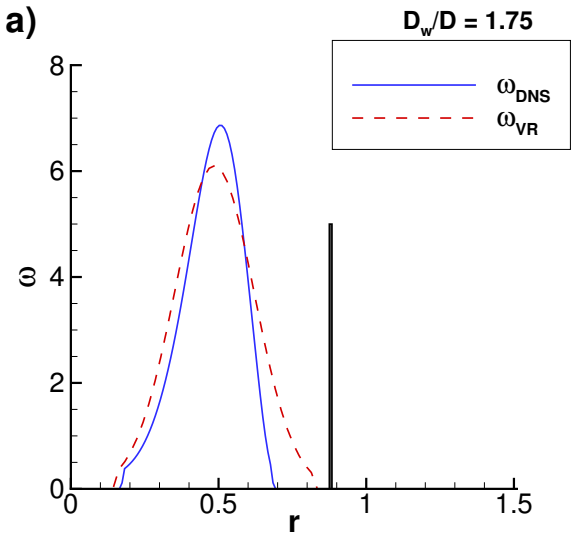

c)

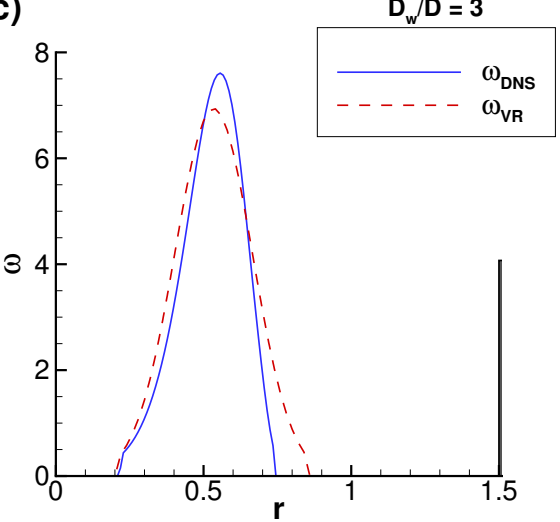

b)

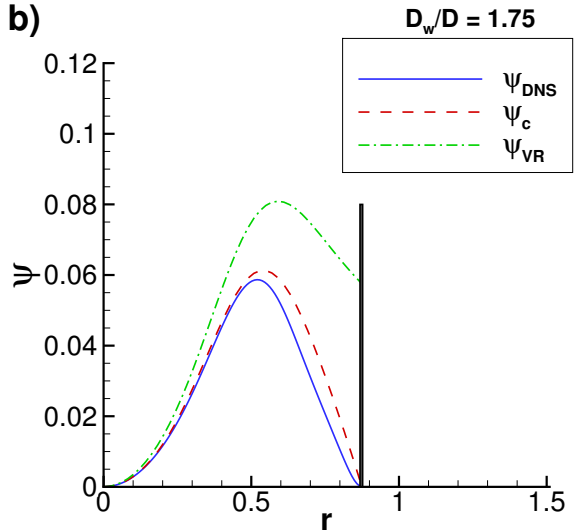

d)

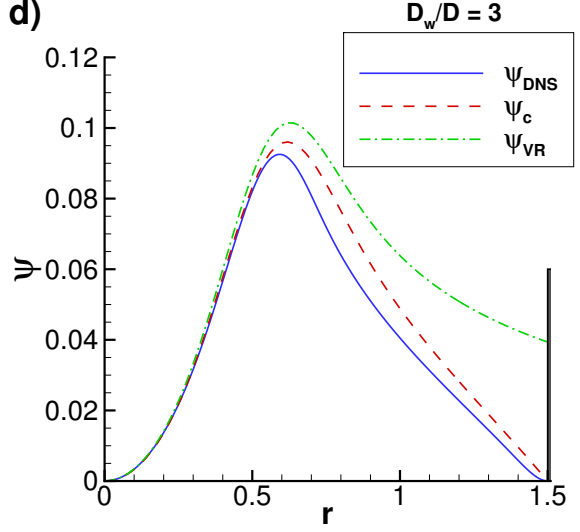

FIGURE 13. Comparison between the DNS data (blue solid curves) and predictions of the vortex ring model (red dashed curves). Profiles are shown along the $r$-axis through the centre of the vortex (at $x=X_{c}$ ). Vorticity $\omega$ and streamfunction $\psi$ profiles are shown for two confinements $D_{w} / D=1.75$ and $D_{w} / D=3$. Streamfunction profiles $\left(\Psi_{\mathrm{VR}}\right)$ of the unconfined vortex ring (formula 2.20) are shown (green dash-dot curves) for reference in figures (b) and (d). Vertical lines indicate the lateral wall. $R e_{\mathrm{D}}=1700, \tau=8$.

also guarantee an accurate representation of the vorticity field, which is not the case in figures 12 (a) and (b). In order to explore this feature, we present in figure 12 (c) a scatter plot of $\omega / r$ as a function of $\Psi$ for points inside the vortex ring core. We have plotted normalised values of vorticity and streamfunction, as in Sullivan (1973), using the vortex ring radius $R_{0}$ and the translation velocity $U$ as reference scales. As one can see from this figure, $\omega / r$ cannot be represented as a simple function of $\psi$, either for the model, or for the DNS vortex. Similar scatter plots were reported in experimental studies of unconfined vortex rings (Sullivan 1973; Akhmetov 2009). The fact that our model is based on the Kaplanski-Rudi model, including viscous and unsteady effects (see $§ 2.3$ and equation 2.24), allows us to obtain a scatter plot which is very close to that obtained by DNS.

To explore the accuracy of our model, we showed in figures 13 and 14 vorticity and streamline profiles through the centre of the vortex. Profiles along the $r$-axis are the most representative, since the presence of the wall at $r=R_{w}$ must be taken into account. Two confinement ratios are considered: $D_{w} / D=1.75$ and $D_{w} / D=3$. Vorticity profiles (figures 13 (a) and (c)) are well approximated by the model, even though the theoretical 
a)

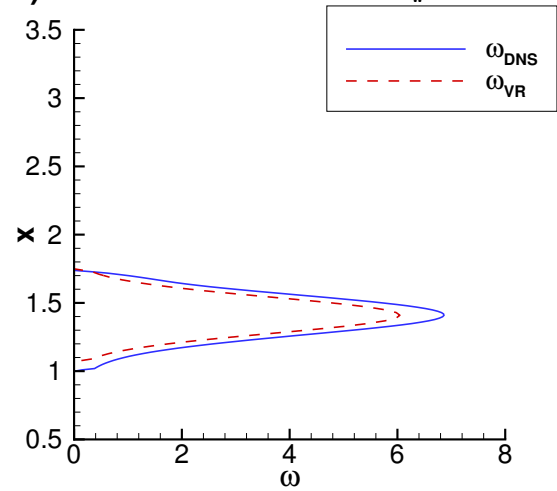

c)

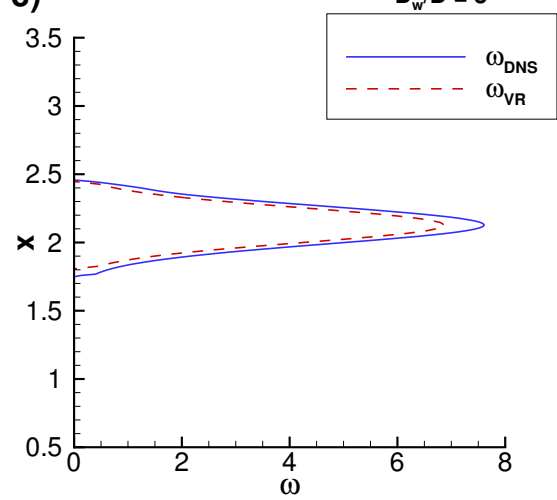

b)

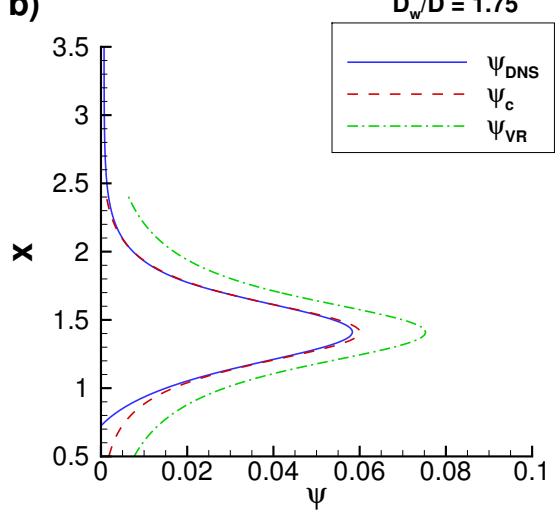

d)

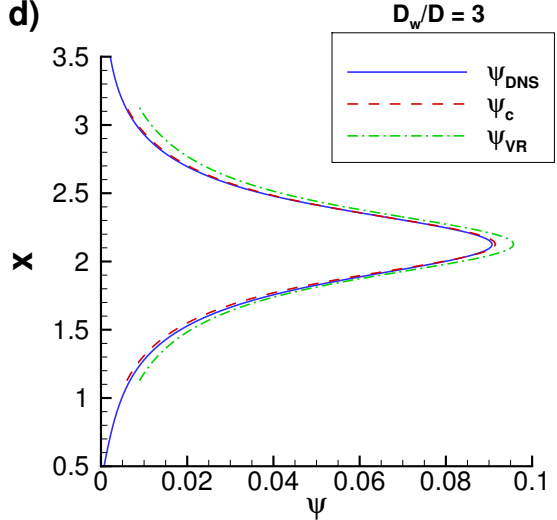

FiguRE 14. The same as in figure 13 , but for the profiles along the $x$-axis through the centre of the vortex (at $\left.r=R_{0}\right)$.

vorticity (see equation 2.16 and its approximation 4.1 by an isotropic two-dimensional Gaussian) does not reproduce the asymmetry of the DNS vorticity profile with respect to the centre of the vortex. We recall that the Gaussian approximation (4.1) stands for relatively thin vortex rings, with small diffusive scales $L$. The asymmetry of vorticity profiles is less important along the longitudinal $x$-axis (figures 14 (a) and (c)), making the theoretical predictions very accurate. In figures 13 (b) and (d), in addition to the streamline profile $\left(\Psi_{c}\right)$ of the confined vortex ring (see equation 2.29) we also plot the streamline profile $\left(\Psi_{\mathrm{VR}}\right)$ of the unconfined vortex ring (see equation 2.27 ). It can be clearly seen from these figures that the correction $\Psi_{0}$ added in (2.29) plays an important role in bringing $\Psi_{c}$ to zero at the wall. We recall that $\mathcal{L} \Psi_{0}=0$ and thus it does not affect the vorticity distribution of the vortex ring. The correction is most visible in the vicinity of the wall $\left(R_{0}<r<R_{w}\right)$ and allows us to obtain a very good approximation of the DNS profiles. We also note that, as expected, the correction is most important for the low confinement ratio $\left(D_{w} / D\right)$ since in this case the wall is at its closest to the vortex centre. In the longitudinal $x$-direction (figures 14 (b) and (d)), the correction due to the wall is less important since the unconfined vortex ring model already gives a good approximation $\left(\Psi_{\mathrm{VR}} \approx \Psi_{c}\right)$.

Note that the quasi-superposition of streamlines implies that the DNS and theoretical vortex rings have similar signatures. The signature of a vortex ring without swirl is a 


\begin{tabular}{|c|c|c|c|c|c|}
\hline \multirow{2}{*}{$\begin{array}{l}D_{w} / D=1.75 \\
\tau_{f}=8\end{array}$} & \multicolumn{3}{|c|}{ model/DNS parameters } & \multicolumn{2}{|c|}{ computed integrals } \\
\hline & $R_{0 f}\left|X_{c f}\right|$ & $L$ & $\theta$ & $\left|\Gamma_{f}\right|$ & $E_{f}$ \\
\hline VR model & $0.503|1.409|$ & 0.132 & 3.794 & |0.687 | - - & 0.0888 \\
\hline DNS data & $0.503|1.415|$ & - & - & $|0.667|$ & 0.0888 \\
\hline \multirow{2}{*}{$\begin{array}{l}D_{w} / D=2.00 \\
\tau_{f}=8\end{array}$} & \multicolumn{3}{|c|}{ model/DNS parameters } & \multicolumn{2}{|c|}{ computed integrals } \\
\hline & $R_{0 f}\left|X_{c f}\right|$ & $L$ & & $\Gamma_{f}$ & $E_{f}$ \\
\hline VR model & $0.535|1.678|$ & $|0.130|$ & 4.099 & | $0.714 \mid$ & 0.1190 \\
\hline DNS data & $0.545|1.675|$ & 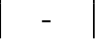 & - & $|0.695|$ & 0.1169 \\
\hline$D_{w} / D=3.00 \|$ & \multicolumn{3}{|c|}{ model/DNS parameters } & \multicolumn{2}{|c|}{ computed integrals } \\
\hline$\tau_{f}=8$ & $R_{0 f} \mid X_{c f}$ & $L$ & $\theta$ & $\Gamma_{f}$ & $E_{f}$ \\
\hline VR model & $0.549|2.126|$ & 0.129 & 4.241 & $|0.742|$ & 0.1581 \\
\hline DNS data & $0.555|2.125|$ & - & - & $|0.721|$ & 0.1495 \\
\hline \multirow{2}{*}{$\begin{array}{l}D_{w} / D=8.00 \\
\tau_{f}=8\end{array}$} & \multicolumn{3}{|c|}{ model/DNS parameters } & \multicolumn{2}{|c|}{ |computed integrals } \\
\hline & $R_{0 f}$ & $L$ & $\theta$ & $\Gamma_{f}$ & $E_{f}$ \\
\hline VR & $|0.546| 2.264 \mid$ & $|0.130|$ & 4.204 & $|0.753|$ & 0.1700 \\
\hline DNS data & $|0.555| 2.265 \mid$ & - & - & $0.731 \mid$ & 0.1583 \\
\hline
\end{tabular}

TABLE 1. Parameters of the vortex ring model obtained by a non-linear fit of (4.1) using DNS data and that computed from the original DNS for various confinement ratios $D_{w} / D$. The results of comparison are presented for the vortex centre position $\left(R_{0 f}, X_{c f}\right)$, circulation $\Gamma_{f}$ and energy $E_{f} \cdot R e_{\mathrm{D}}=1700, \tau=8$.

topological invariant, defined by Moffatt (1988) as the volume inside the torus $\psi=c s t$. (see also Danaila \& Helie (2008)).

Table 1 shows the comparison between the predictions of DNS and modelling for three other confinement parameters $D_{w} / D=2,3,8$. For small confinements (large $D_{w} / D$ ) the model tends to overestimate the circulation of the vortex ring, but by not more than $3 \%$. The values of the energy $E_{f}$, computed from equation (4.4) using the fitted vorticity distribution, are in very good agreement with the DNS results for all confinement ratios. This implies that the theoretical model for the streamfunction $\Psi_{c}$ is not only qualitatively accurate (as shown in figure 12), but can also be used for quantitative characteristics of vortex rings.

\subsection{Evolution of integral characteristics for $R e_{D}=1700$}

In the next step, the time evolutions of integral characteristics (circulation, energy and translational velocity), predicted by the model with viscous length given by equation (4.2), are compared with those obtained by DNS. The vorticity field was truncated so that only values $\omega / \omega_{\max } \geqslant 0.05$, representing the vortex core, were considered. Using the fitted vortex ring at $\tau=8$ (with parameters displayed in table 1) we computed model 


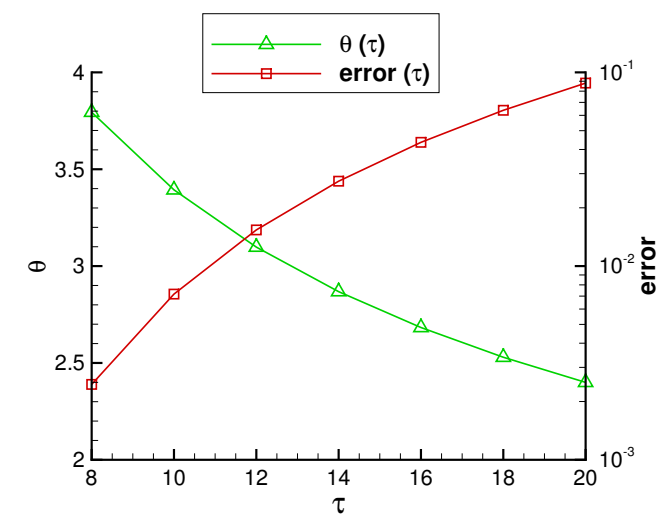

Figure 15. Time evolution of parameter $\theta$ assumed in the model (see equation 2.24) and corresponding maximum relative error intrinsic to the model (see expression 2.30). $R e_{\mathrm{D}}=1700$.

a)

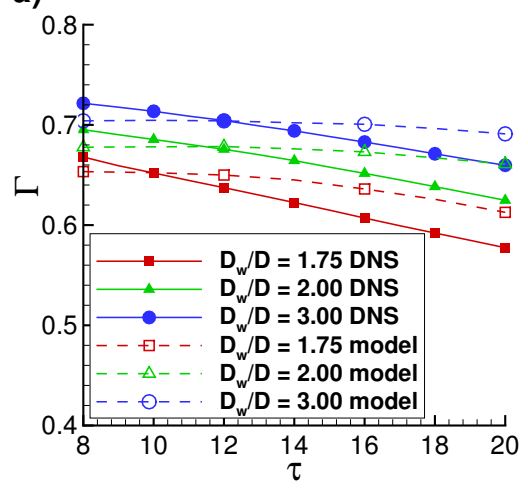

b)

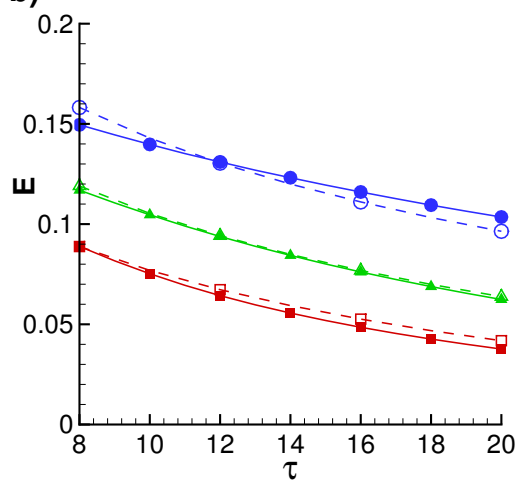

FiguRE 16. Time evolution of the circulation $\Gamma$ (a) and energy $E$ (b) of the vortex ring obtained by DNS and the model for three confinement parameters $D_{w} / D \cdot R e_{\mathrm{D}}=1700$.

predictions corresponding to the time evolution of the viscous scale given by (2.24) for a long time interval $8 \leqslant \tau \leqslant 20$. To check whether this case lies inside the range of applicability of the model (see section 2.3 and figure 3 ), we plotted in figure 15 the time evolution of parameter $\theta$ and the corresponding maximum relative errors of the model (see equation 2.30). As follows from figure 15, the model offers a very good approximation, despite the strong confinement (corresponding to $\varepsilon \approx 1 /(1.75)$ in figure 3 ).

The time evolution of circulation and energy, predicted by the model and DNS for confinement parameters $D_{w} / D=1.75,2$ and 3 , are shown in figure 16 . For the sake of clarity, the plots for $D_{w} / D=8$, which were very close to the plots for the case $D_{w} / D=3$, are not shown in the figure. Remembering equation (2.17), one can expect that the circulation predicted by the model is close to $\Gamma_{f}$ considered at the fitting time $\tau=\tau_{f}$. Figure 16 (a) shows that the rate of decrease of circulation inferred from DNS data is higher than the one predicted by the model. However, theoretical values of $\Gamma$ are very close to DNS values, especially if smaller time intervals are considered. This explains why the time evolutions of energies, inferred from DNS data and predicted by the model (see figure $16(\mathrm{~b})$ ), are close. The idea behind this comparison was to challenge our model 


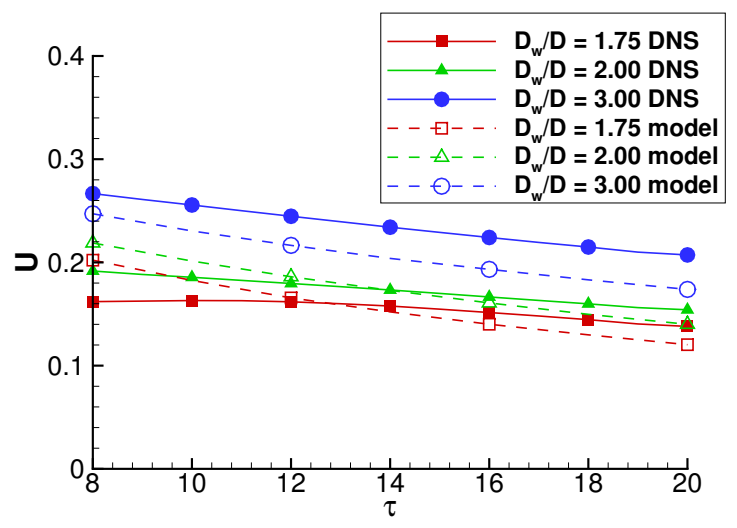

FIgURE 17. Time evolution of the translational velocity $U$ of the vortex ring. Comparison between DNS data and the model predictions (4.5) for three confinement parameters $D_{w} / D$. $R e_{\mathrm{D}}=1700$

to predict the time evolution of integral characteristics for long time intervals. Strictly speaking, this is beyond the capabilities of the model, since the original Kaplanski-Rudi vortex ring model is valid for small time intervals when the Reynolds number is large (Fukumoto \& Kaplanski 2008). However, figure 16 shows that the model could also be applied for large time evolutions, with fairly good accuracy. This opens the way to using the model for realistic configurations of practical interest.

Finally, we compare the theoretical prediction (4.5) for the translational velocity of the vortex ring with the DNS values computed from the time evolution of the vorticity centroid (4.8) (see figure (17)). We recover two of the characteristic features of the vortex ring motion discussed in section 2: the translational velocity $U$ is larger for low confinements (large $D_{w} / D$ ratios) and $U$ decreases faster when the confinement is low. These are two consequences of the interaction of the vortex ring with the vorticity created near the lateral wall (with the sign opposite to the vorticity of the vortex ring). Our model accounts for these features of the confined vortex ring motion, but the values of $U$ are underestimated compared to those obtained by DNS. The origin of these discrepancies is the role of the stream-wise gradient $\partial \Psi_{c}^{*} / \partial x_{1}$ present in formula (4.5); theoretical streamfunction $\Psi_{c}^{*}$ displays larger gradients near the wall than those inferred from the DNS field, resulting in lower values of the translational velocity predicted by the model. The model, however, is able to predict well the main features of the variation of the translational velocity of the confined vortex ring.

\subsection{Evolution of integral characteristics for higher Reynolds numbers}

In an attempt to further challenge our model, we considered the case of a higher Reynolds number $\left(R e_{\mathrm{D}}=3400\right)$ and long time evolution of the vortex ring during the postformation phase. We kept the same injection program (see figure 4) with $\tau_{\text {off }}=2.26$ and focused on $15 \leqslant \tau \leqslant 60$. DNS computations were performed on a larger domain $\left(L_{d}=20\right)$ to account for the long time evolution. We used the same spatial resolution and the same timestep as for previous simulations to ensure mesh convergence of the results. Confinement parameters $D_{w} / D \geqslant 2$ were considered in order to avoid the situation where the vortex ring touches the lateral wall during long time evolutions. Figure (18) shows a comparison between the values of normalised vorticity and streamfunction predicted by the model and DNS at $\tau=15$ and $D_{w} / D=2$. As one can see in this figure, 
a)

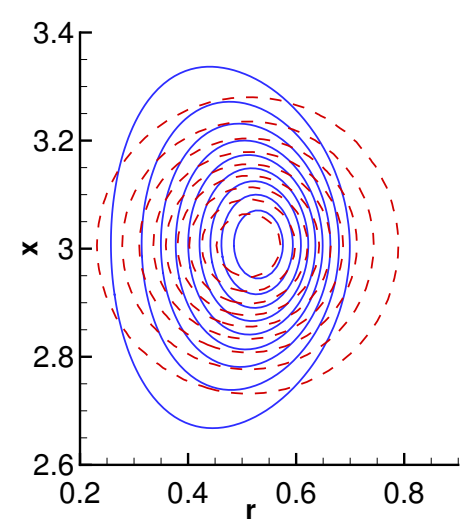

b)

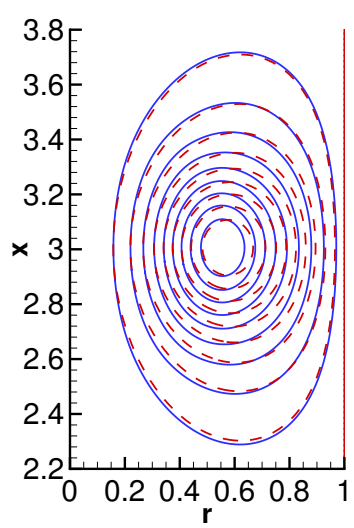

FIGURE 18. Contours of normalised vorticity $\omega / \omega_{\max }$ (a) and corresponding normalised streamfunction $\psi / \psi_{\max }$ (b), obtained by DNS (blue solid curves) and the vortex ring model (red dashed curves). The values of $\omega / \omega_{\max }$ and $\psi / \psi_{\max }$ are shown from 0.1 to 0.9 with increments of 0.1 . $R e_{\mathrm{D}}=3400, D_{w} / D=2, \tau=15$.
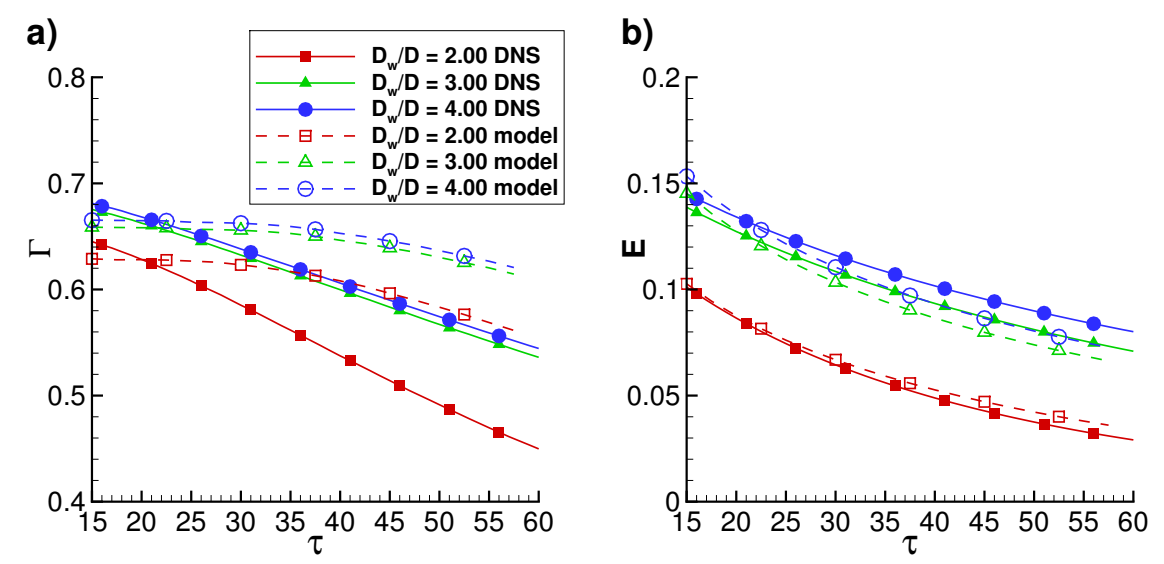

Figure 19. Time evolution of the circulation $\Gamma$ (a) and energy $E$ (b) of the vortex ring, as inferred from DNS data and predicted by the model for three confinement parameters $D_{w} / D$. $R e_{\mathrm{D}}=3400$.

the time evolution trends for both parameters, predicted by the model and obtained by DNS, are rather similar. This means that the two vortex rings have the same signature, despite rather different spatial distributions of the vorticity. Table 2 summarises the predictions of the main parameters defining the vortex rings for various confinement parameters $D_{w} / D$. The spatial positions of the vortex are accurately captured by the model, whilst it slightly overestimates the initial circulation. The time evolutions of the vortex ring circulation and energy are compared in figure 19. We notice in this figure the same trends as for $R e_{\mathrm{D}}=1700$ : the theoretical rate of circulation decay is lower than inferred from DNS data, while the energy predictions made by the model are very close to numerical results. 


\begin{tabular}{|c|c|c|c|c|c|}
\hline \multirow{2}{*}{$\begin{array}{l}D_{w} / D=2 \\
\tau_{f}=15\end{array}$} & \multicolumn{3}{|c|}{ model/DNS parameters } & \multicolumn{2}{|c|}{ computed integrals } \\
\hline & $R_{0 f} \mid X_{c f}$ & $L$ & $\mid \theta$ & $\Gamma_{f}$ & $E_{f}$ \\
\hline VR model & $|0.527| 3.006 \mid$ & 0.1275 & 4.135 & $5 \mid 0.6619$ & 0.102 \\
\hline DNS data & $|0.535| 3.005 \mid$ & - & - & 0.6443 & 0.101 \\
\hline$D_{w} / D=3$ & model/DNS & parame & eters & compute & d integrals \\
\hline$\tau_{f}=15$ & $R_{0 f}\left|X_{c f}\right|$ & $L$ & $\theta$ & $\left|\Gamma_{f}\right|$ & $E_{f}$ \\
\hline VR model & $|0.556| 3.937 \mid$ & $0.125 \mid$ & 4.438 & | $0.693 \mid$ & 0.1452 \\
\hline DNS data & $0.565|3.935|$ & - & - & 0.674 & 0.1387 \\
\hline$D_{w} / D=4$ & model/DNS & parame & eters & compute & d integrals \\
\hline$\tau_{f}=15$ & $R_{0 f} \mid X_{c f}$ & $L$ & $\theta$ & $\Gamma_{f}$ & $E_{f}$ \\
\hline VR model & $0.556|4.157|$ & 0.125 & 4.435 & 0.700 & 0.1532 \\
\hline DNS data & $0.565|4.155|$ & - & - & 0.681 & 0.1450 \\
\hline
\end{tabular}

TABLE 2. Parameters of the vortex ring model obtained by a non-linear fit of (4.1) using DNS data and that computed from the original DNS for various confinement ratios $D_{w} / D$. The results of comparison are presented for the vortex centre position $\left(R_{0 f}, X_{c f}\right)$, circulation $\Gamma_{f}$ and energy $E_{f} . R e_{\mathrm{D}}=3400, \tau=15$.

\section{Summary and discussion}

A new theoretical model for a confined axisymmetric vortex ring is suggested. This model allows us, for the first time to the best of our knowledge, to provide a good fit with realistic vortex rings generated in experiments and those obtained by direct numerical simulations (DNS). The latter were used to test the capabilities of the model and to investigate new features of the confined vortex rings reported in the experimental study conducted by Stewart et al. (2012).

The model combines the viscous vortex ring model by Kaplanski and Rudi (Kaplanski \& Rudi 2005) with Brasseur's (Brasseur 1979) approach to derive a wall-induced streamfunction correction. A closed relationship for the streamfunction of the confined vortex ring is obtained. The vorticity field $\omega(r, x)$ predicted by the model is shown to have the same trends as the one obtained by DNS, while the spatial distribution of the streamfunction $\psi(r, x)$ predicted by the model is shown to be very close to the one obtained by DNS. This allows us to estimate accurately two important characteristics of the confined vortex ring depending only on the streamfunction: its signature $V(\psi)=\{$ the volume inside the torus $\psi=c s t$. $\}$, defined by Moffatt (1988) as a topological invariant which enables us to identify the vortex ring, and the vortex ring energy $E=\pi \iint \omega \psi d r d x=\pi \iint \frac{1}{r}\left[(\partial \psi / \partial r)^{2}+(\partial \psi / \partial x)^{2}\right] d r d x$

Making the assumption that there is a power-law variation in the viscous length of the vortex ring (see expression (4.2)), the time variations of the main integral characteristics, circulation $\Gamma$, kinetic energy $E$ and translational velocity $U$, are obtained. The predictions of the model have been compared with DNS data for various Reynolds numbers and 
confinement parameters. Even for high Reynolds numbers and long time evolutions of the confined vortex ring in the post-formation phase (the most unfavourable conditions), the model offered good approximation of the DNS data. For example, variations in energy over time were accurately captured, while circulation decay and the evolution of the translational velocity were predicted fairly well, which enables us to recommend the model for practical engineering applications. The fit with DNS data could be further improved if a power different to $1 / 2$ was considered in expression (4.2) was considered; it was earlier shown that the Kaplanski-Rudi model for viscous vortex ring could be generalised for power-laws with exponents between 0.25 and 0.5 (see Kaplanski et al. (2009)). The main aim of this paper, however, is not to find the best fit with DNS data but to assess the general capabilities of the model to reproduce realistic confined vortex rings.

It is shown that another way to improve the model is to consider a vortex ring taking into account the ellipticity (deformation) of the vorticity field (as shown in figures 16 and 18). This would allow us to describe more accurately the circulation decay of the confined vortex ring, since its rate of decay strongly depends on its confinement (as can be inferred from our DNS results and was observed in experiments by Stewart et al. (2012)). Such an improved model could be applied for the estimate of the formation number (Gharib et al. 1998) of a confined vortex ring, using the criteria introduced by Shusser \& Gharib (2000) or Mohseni \& Gharib (1998).

The authors are grateful to EPSRC (grants EP/K005758/1 and EP/M002608/1) for the financial support of this project.

\section{REFERENCES}

Akнmetov, D. G. 2009 Vortex Rings. Berlin, Heidelberg: Springer-Verlag.

Amick, C. J. \& Fraenkel, L. E. 1986 The uniqueness of Hill's spherical vortex. Arch. Rational Mech. Anal. 92, 91-119.

Amick, C. J. \& Fraenkel, L. E. 1988 The uniqueness of a family of vortex rings. Arch. Rational Mech. Anal. 3, 207-241.

Batchelor, G. K. 1988 An Introduction to Fluid Dynamics, 7th edn. Cambridge, New York: Cambridge University Press.

Begg, S., Kaplanski, F., Sazhin, S., Hindle, M. \& Heikal, M. 2009 Vortex ring-like structures in gasoline fuel sprays under cold-start conditions. International Journal of Engine Research 10 (4), 195-214.

Brasseur, J. G. 1979 Kinematics and dynamics of vortex rings in a tube. Ph.D. Thesis, report JIAA, TR-26, Joint Institute for Aeronautics and Acoustics, Dept. of Aeronautics and Astronautics, Stanford University.

Cater, J.E., Soria, J. \& Lim, T.T. 2004 The interaction of the piston vortex with a pistongenerated vortex ring. J. Fluid Mech. 499, 327-343.

DABIRI, J. O. 2009 Optimal vortex formation as a unifying principle in biological propulsion. Annual Review of Fluid Mechanics 41 (1), 17-33.

Dabiri, J. O. \& Gharib, M. 2004 Fluid entrainment by isolated vortex rings. J. Fluid Mech. $\mathbf{5 1 1}, 311-331$.

DABiRi, J. O. \& Gharib, M. 2005 Starting flow through nozzles with temporally variable exit diameter. J. Fluid Mech. 538, 111-136.

DANAila, I. \& Helie, J. 2008 Numerical simulation of the postformation evolution of a laminar vortex ring. Phys. Fluids 20, 073602.

Danaila, I., Vadean, C. \& Danaila, S. 2009 Specified discharge velocity models for the numerical simulation of laminar vortex rings. Theor. Comput. Fluid Dynamics 23, 317332.

EsteBAn, M. J. 1983 Nonlinear elliptic problems in strip-like domains: symmetry of positive vortex rings. Nonlinear Analysis, Theory, Methods \& Applications 7, 365-379. 
Fraenkel, L. E. \& Berger, M. S. 1974 A global theory of steady vortex rings in an ideal fluid. Acta Math. 132, 13-51.

Fukumoto, Y. 2010 Global evolution of viscous vortex rings. Theor. Comput. Fluid Dynamics 24, 335-347.

Fukumoto, Y. \& Kaplanski, F. B. 2008 Global time evolution of an axisymmetric vortex ring at low Reynolds numbers. Phys. Fluids 20, 053103.

Fukumoto, Y. \& Moffatt, H. K. 2000 Motion and expansion of a viscous vortex ring. part 1. a higher-order asymptotic formula for the velocity. J. Fluid Mech. 417, 1-45.

Fukumoto, Y. \& Moffatt, H. K. 2008 Kinematic variational principle for motion of vortex rings. Physica D 237, 2210-2217.

Gharib, M., Rambod, E., Kheradvar, A., Sahn, D.J. \& Dabiri, J.O. 2006 Optimal vortex formation as an index of cardiac health. Proceedings of the National Academy of Sciences (PNAS) 103, 6305-6308.

Gharib, M., RAmbod, E. \& Shariff, K. 1998 A universal time scale for vortex ring formation. J. Fluid Mech. 360, 121-140.

Hecht, F. 2012 New developments in Freefem++. Journal of Numerical Mathematics 20, 251266.

Hecht, F., Pironneau, O., Hyaric, A. Le \& Ohtsuke, K. 2007 FreeFem++ (manual). www.freefem.org.

Helmholtz, H. 1958 On integrals of the hydrodynamical equations which express vortexmotion. Crelle's J. 55, 485-512.

Hill, M. J. M. 1894 On a spherical vortex. Philos. Trans. Roy. Soc. London A185, 213-245.

James, S. \& Madnia, C. K. 1996 Direct numerical simulation of a laminar vortex ring. Phys. Fluids 8, 2400-2414.

Kaplanski, F., Sazhin, S. S., Begg, S., Fukumoto, Y. \& Heikal, M. 2010 Dynamics of vortex rings and spray-induced vortex ring-like structures. European Journal of Mechanics B/Fluids 29 (3), 208-216.

Kaplanski, F., Sazhin, S. S., Fukumoto, Y., Begg, S. \& Heikal, M. 2009 A generalized vortex ring model. J. Fluid Mech. 622, 233-258.

Kaplanski, F. B., Fukumoto, Y. \& Rudi, Y. A. 2012 Reynolds-number effect on vortex ring evolution in a viscous fluid. Phys. Fluids 24, 033101.

Kaplanski, F. B. \& Rudi, Y. A. 1999 Dynamics of a viscous vortex ring. Int. J. Fluid Mech. Res. 26, 618-630.

Kaplanski, F. B. \& Rudi, Y. A. 2005 A model for the formation of "optimal" vortex ring taking into account viscosity. Phys. Fluids 17, 087101-087107.

Kim, J. \& Moin, P. 1985 Application of a fractional step method to incompressible NavierStokes equations. J. Comput. Physics 59, 308-323.

Krieg, M. \& Mohseni, K. 2013 On the approximation of the translational velocity of vortex rings. J. Fluids Engineering 135, 124501.

Krueger, P. S. 2008 Circulation and trajectories of vortex rings formed from tube and orifice openings. Physica D 237, 2218-2222.

Krueger, S. \& Gharib, M. 2003 The significance of vortex ring formation on the impulse and thrust of a starting jet. Phys. Fluids 15, 1271-1280.

Lamb, H. 1932 Hydrodynamics. Dover, New York.

Lim, T. T. \& Nickels, T. B. 1995 Vortex rings, in Vortices in Fluid Flows, edited by S. I. Green, Kluwer, Dordrecht, 1995, p. 95.

Linden, P. F. \& Turner, J. S. 2001 The formation of 'optimal' vortex rings, and the efficiency of propulsion devices. J. Fluid Mech. 427, 61-72.

Mathematica 2007 MATHEMATICA Book. version 6.0.0, http://functions.wolfram.com Wolfram Research Inc.

Michalke, A. 1984 Survey on jet instability theory. Prog. Aerospace Sci. 21, 159-199.

Moffatt, H. K. 1988 Generalised vortex rings with and without swirl. Fluid Dyn. Res. 3, $22-30$.

Mohseni, K. 2001 Statistical equilibrium theory for axisymmetric flow: Kelvin's variational principle and an explanation for the vortex ring pinch-off process. Phys. Fluids 13, 1924.

Mohseni, K. 2006 A formulation for calculating the translational velocity of a vortex ring or pair. Bioinspiration \& Biomimetics 1, S57-S64. 
Mohseni, K. \& Gharib, M. 1998 A model for universal time scale of vortex ring formation. Phys. Fluids 10, 2436-2438.

Ni, W. M. 1980 On the existence of global vortex rings. J. d'Analyse Math. 37, 208-247.

Norbury, J. 1972 A steady vortex ring close to Hill's spherical vortex. Proc. Cambridge Phil. Soc. 72, 253-282.

Norbury, J. 1973 A family of steady vortex rings. J. Fluid Mech. 57, 417-431.

Orland, P. 1999 Fluid Flow Phenomena: A Numerical Toolkit. Dordrecht: Kluwer Academic Publishers.

Orlanski, I. 1976 A simple boundary condition for unbounded hyperbolic flows. J. Comput. Physics 21, 251-269.

Phillips, O. M. 1956 The final period of decay of non-homogeneous turbulence. Proc. Cambridge Phil. Soc. 252, 135-151.

RAI, M. \& Moin, P. 1991 Direct simulations of turbulent flow using finite-difference schemes. J. Comput. Physics 96, 15-53.

Rosenfeld, M., Rambod, E. \& Gharib, M. 1998 Circulation and formation number of a laminar vortex ring. J. Fluid Mech. 376, 297-318.

Rott, N. \& Cantwell, B. $1993 a$ Vortex drift. i: Dynamic interpretation. Phys. Fluids 5, $1443-1450$.

Rott, N. \& CAntwell, B. $1993 b$ Vortex drift. ii: The flow potential surrounding a drifting vortical region. Phys. Fluids 5, 1451-1455.

Ruith, M. R., Chen, P. \& Meiburg, E. 2004 Development of boundary conditions for direct numerical simulations of three-dimensional vortex breakdown phenomena in semi-infinite domains. Computers \& Fluids 33, 1225-1250.

Saffman, P. G. 1970 The velocity of viscous vortex rings. Studies in Applied Math. 49, 371-380.

Saffman, P. G. 1992 Vortex Dynamics. Cambridge, New York: Cambridge University Press.

SAU, R. \& MAhesh, K. 2007 Passive scalar mixing in vortex rings. J. Fluid Mech. 582, 449-461.

SazHin, S. S. 2014 Droplets and Sprays. London, Heidelberg: Springer.

Shariff, K. \& Leonard, A. 1992 Vortex rings. Ann. Rev. Fluid Mech. 24, 235-279.

Shusser, M. \& Gharib, M. 2000 Energy and velocity of a forming vortex ring. Phys. Fluids 12, 618-621.

Stewart, K., Niebel, C., Jung, S. \& Vlachos, P. 2012 The decay of confined vortex rings. Experiments in Fluids 53, 163-171.

Sullivan, I. S., Niemela, J. J., Hershberger, R. E. Bolster, D. \& Donnelly, R. J. 2008 Dynamics of thin vortex rings. J. Fluid Mech. 609, 319-347.

Sullivan, J. P. 1973 Study of a vortex ring using a laser doppler velocimeter. AIAA Journal 11, 1384.

Tung, C. \& Ting, L. 1967 Motion and decay of a vortex ring. Phys. Fluids 10, 901-910.

Verzicco, R. \& Orlandi, P. 1996 A finite-difference scheme for three-dimensional incompressible flow in cylindrical coordinates. J. Comput. Physics 123, 402-414.

Weigand, A. \& Gharib, M. 1997 On the evolution of laminar vortex rings. Exp. Fluids 22, 447-457.

Zhao, W., Steven, H. F. \& Mongeau, L. G. 2000 Effects of trailing jet instability on vortex ring formation. Phys. Fluids 12, 589-596. 